\title{
Pathways of topological rank analysis (PoTRA): a novel method to detect pathways involved in hepatocellular carcinoma
}

\author{
Chaoxing Li ${ }^{\text {Corresp., }}{ }^{1}, \mathbf{L i ~ L i u}^{2}{ }^{2}$, Valentin Dinu ${ }^{2}$ \\ ${ }^{1}$ School of Life Sciences, Arizona State University, Tempe, Arizona, United States \\ 2 Department of Biomedical Informatics, Arizona State University, Scottsdale, Arizona, United States \\ Corresponding Author: Chaoxing Li \\ Email address: chaoxing@asu.edu
}

Complex diseases such as cancer are usually the result of a combination of environmental factors and one or several biological pathways consisting of sets of genes. Each biological pathway exerts its function by delivering signaling through the gene network.

Theoretically, a pathway is supposed to have a robust topological structure under normal physiological conditions. However, the pathway's topological structure could be altered under some pathological condition. It is well known that a normal biological network includes a small number of well-connected hub nodes and a large number of nodes that are non-hubs. In addition, it is reported that the loss of connectivity is a common topological trait of cancer networks, which is an assumption of our method. Hence, from normal to cancer, the process of the network losing connectivity might be the process of disrupting the structure of the network, namely, the number of hub genes might be altered in cancer compared to that in normal or the distribution of topological ranks of genes might be altered. Based on this, we propose a new PageRank-based method called Pathways of Topological Rank Analysis (PoTRA) to detect pathways involved in cancer. We use PageRank to measure the relative topological ranks of genes in each biological pathway, then select hub genes for each pathway, and use Fisher's exact test to test if the number of hub genes in each pathway is altered from normal to cancer. Alternatively, if the distribution of topological ranks of gene in a pathway is altered between normal and cancer, this pathway might also be involved in cancer. Hence, we use the KolmogorovSmirnov test to detect pathways that have an altered distribution of topological ranks of genes between two phenotypes. We apply PoTRA to study hepatocellular carcinoma (HCC) and several subtypes of HCC. Very interestingly, we discover that all significant pathways in HCC are cancer-associated generally, while several significant pathways in subtypes of HCC are HCC subtype-associated specifically. In conclusion, PoTRA is a new approach to explore and discover pathways involved in cancer. PoTRA can be used as a complement to 
other existing methods to broaden our understanding of the biological mechanisms behind cancer at the system-level. 
1 Pathways of topological rank analysis (PoTRA): a novel method to detect pathways

2 involved in hepatocellular carcinoma

3 Chaoxing $\mathrm{Li}^{1 *}, \mathrm{Li} \mathrm{Liu}^{2}$, Valentin Dinu ${ }^{2}$

$4{ }^{1}$ School of Life Sciences, Arizona State University, Tempe, AZ 85287, USA

$5 \quad{ }^{2}$ Department of Biomedical Informatics, Arizona State University, Scottsdale, AZ 85255, USA

6

7 Corresponding Author:

8 Chaoxing Li

9

10 Email address: chaoxing@asu.edu

11

12

13

14

15

16

17

18

19

20

21

22

23

24

25

26

27

28

29

30

31

32 Abstract 
Complex diseases such as cancer are usually the result of a combination of environmental factors and one or several biological pathways consisting of sets of genes. Each biological pathway exerts its function by delivering signaling through the gene network. Theoretically, a pathway is supposed to have a robust topological structure under normal physiological conditions. However, the pathway's topological structure could be altered under some pathological condition. It is well known that a normal biological network includes a small number of well-connected hub nodes and a large number of nodes that are non-hubs. In addition, it is reported that the loss of connectivity is a common topological trait of cancer networks, which is an assumption of our method. Hence, from normal to cancer, the process of the network losing connectivity might be the process of disrupting the structure of the network, namely, the number of hub genes might be altered in cancer compared to that in normal or the distribution of topological ranks of genes might be altered. Based on this, we propose a new PageRank-based method called Pathways of Topological Rank Analysis (PoTRA) to detect pathways involved in cancer. We use PageRank to measure the relative topological ranks of genes in each biological pathway, then select hub genes for each pathway, and use Fisher's exact test to test if the number of hub genes in each pathway is altered from normal to cancer. Alternatively, if the distribution of topological ranks of gene in a pathway is altered between normal and cancer, this pathway might also be involved in cancer. Hence, we use the Kolmogorov-Smirnov test to detect pathways that have an altered distribution of topological ranks of genes between two phenotypes. We apply PoTRA to study hepatocellular carcinoma (HCC) and several subtypes of HCC. Very interestingly, we discover that all significant pathways in HCC are cancer-associated generally, while several significant pathways in subtypes of HCC are HCC subtype-associated specifically. In conclusion, PoTRA is a new approach to explore and discover pathways involved in cancer. PoTRA can be used as a complement to other existing methods to broaden our understanding of the biological mechanisms behind cancer at the system-level.

\section{Introduction}

High throughput technologies, such as genomic sequencing and microarrays, allow the genomewide analysis of molecular factors associated with disease. While the technologies have advanced and have been refined to generate an increasing amount of high quality data, challenges remain in understanding the biological processes involved in disease etiology, particularly for complex disorders.

As we know, individual genomic alterations may result in diseases. For example, Hemophilia A is an X-linked recessive bleeding disorder caused by a deficiency in the activity of coagulation factor VIII (Franchini \& Mannucci, 2012). Huntington disease (HD) is an autosomal dominant progressive neurodegenerative disorder with a distinct phenotype characterized by chorea, dystonia, incoordination, cognitive decline, and behavioral difficulties, which is caused by a heterozygous expanded trinucleotide repeat (CAG)n, encoding glutamine, in the gene encoding huntingtin (HTT) on chromosome 4p16 (Walker, 2007; Dayalu \& Albin, 2015).

In addition to monogenic diseases, many diseases are complex, such as diabetes, schizophrenia, or cancer, and are believed to be caused by a combination of genomic alterations, epigenetic, environmental and lifestyle factors (Schork, 1997; Hindorff, Gillanders \& Manolio, 2011). 
Genomic disease association analysis suggests that complex diseases are not caused by individual genomic alterations. First, the complex disease phenotype is associated with many genes. Second, it may be associated with interactions among many genes. Therefore, more and more literature has been focusing on analyzing sets of genes associated with some phenotype. Gene expression profiles have been used to assess the activity of biological networks. Several approaches have been developed to identify active subnetworks across different phenotypes from changes in gene expression. One of the first such studies is a general approach to searching for "active sub-networks" associated with high levels of differential expression (Ideker et al., 2002). This approach identifies a set of genes that form a subnetwork whose expression is altered across two different phenotypes. Another very well-known method, Gene Set Enrichment Analysis (GSEA) (Subramanian et al., 2005), is a computational method that determines whether a predefined set of genes shows statistically significant, concordant differences between two phenotypes, which is also based on differential expression of a set of genes between two phenotypes. These approaches, while powerful and popular, are limited by the fact that they ignore the topology of the gene networks and sets that they investigate. Indeed, differential gene expression level in biological network might influence phenotypes. However, only investigating the differential expression levels of gene may be not sufficient since the topology of biological pathway is also an important characteristic of biological pathways and the role they play in both normal and pathological processes, as described below.

It is well known that the topological structure is very important for biological networks and it determines how genes interact with each other, governing how specific genes and biological pathways operate in the promotion or inhibition of human diseases (Tavazoie et al., 1999; Goeman \& Bühlmann, 2007; Tarca et al., 2009; Taylor et al., 2009; Khatri, Sirota \& Butte, 2012; Rhinn et al., 2013; Mitrea et al., 2013). Related to this, a hub gene within a biological network is an important gene which acts to influence the activity of a number of genes (Flintoft, 2004), even influence the activity and function of the entire biological network. Hence, there has been an increased interest to analyze the co-regulation and co-expression of genes in the biological network, and many approaches have been developed to identify differential co-regulation and coexpression of genes in the subnetwork (Kostka \& Spang, 2004; Lai et al., 2004; Reverter et al., 2006; Watson, 2006; Choi \& Kendziorski, 2009; Leonardson et al., 2010; Langfelder et al., 2011; Odibat \& Reddy, 2012). It has been a trend to extend differential expression analysis to differential network analysis (de la Fuente, 2010).

Most of the approaches for topology-based network and pathway analysis are based on different correlation-based metrics to identify differential networks between two different phenotypes. Generally, there are three main ways to compare networks for differential network analysis. The first approach handles weighted networks and uses some functions of the edge-specific weight differences as edge weights to construct differential networks (Hudson, Reverter \& Dalrymple, 2009; Tesson, Breitling \& Jansen, 2010; Liu et al., 2010; Rhinn et al., 2013). The second approach tries to find co-expressed gene sets and identify which correlation patterns are different between sets across conditions (Watson, 2006; Rahmatallah, Emmert-Streib \& Glazko, 2014). This approach formulates summary measures that represent co-expression in a biological network and compares the metric between sets. The third approach compares the topology of 
117 biological networks across different phenotypes by using measures such as degree of nodes or 118 modularity (Reverter et al., 2006; Zhang et al., 2009). However, the PoTRA method uses a

119

120

121

122

123

124

125

126

127

128

129

130

131

132

133

134

135

136

137

138

139

140

141

142

143

144

145

146

147

148

149

150

151

152

153

154

155

156

157

158 topology-based metric to identify differential networks between two phenotypes. In addition to using different metrics, some of the other tools are based on correlation pattern of genes and identify groups of genes whose correlation patterns behave differentially across different datasets (Watson, 2006; Hudson, Reverter \& Dalrymple, 2009; Tesson, Breitling \& Jansen, 2010; Liu et al., 2010; Rhinn et al., 2013). Compared to these tools, PoTRA is directly based on topological ranks of genes and aims to identify pathways where the topological ranks of genes are different across datasets, which is more biologically intuitive. In this method, not only do we use correlation networks but we also use combined networks by taking intersected networks of correlation networks and KEGG curated pathways. Hence, when KEGG curated pathway information is employed, the topological rank-based PoTRA method can apply to the combined networks, while the previous correlation-based methods cannot, which is a limitation of the previously discussed correlation-only based methods. Regarding the previous tools based on topology (Reverter et al., 2006; Zhang et al., 2009), Zhang et al. focuses on identifying genes involved in topological changes, while PoTRA focuses on identifying pathways involved in topological changes. Also, Reverter et al. focuses on identifying genes with differential connectivity between two phenotypes, which is also different from PoTRA's application scenario.

Although the above methods for differential network analysis can deal with some important biological questions, they are still limited. In general, they are based on a basic hypothesis that some connections between genes across the groups could be thought of as "passenger" events and other connections are unique to either one of groups and thus could be "driver" events that contribute to disease progression and development. Hence, they focus on the contribution of individual differential connections to disease. This results in several limitations. First, each differential connection is regarded by these methods to have an equal contribution to disease. However, it is well understood that loss of a connection between two hub genes from normal to disease is more deleterious than loss of a connection between two non-hub genes. Second, how differential connections ("driver" connections mentioned above) between pairs of genes are associated with diseases is still not very biologically intuitive, because how the dependency between genes contributes to diseases is usually little understood.

To address these problems, we propose a new PageRank-based method called Pathways of Topological Rank Analysis (PoTRA) to detect pathways associated with cancer. PageRank is an algorithm initially used by Google Search to rank websites in their search engine results (Page et al., 1999). It is a way of measuring the importance of nodes in a network. More generally, PageRank has been applied to other networks, e.g., social networks (Pedroche et al., 2013; Wang et al., 2013). To date, there have been several studies using PageRank for gene expression and network analysis (Morrison et al., 2005; Winter et al., 2012; Kimmel \& Visweswaran, 2013; Hou \& Ma, 2014; Bourdakou, Athanasiadis \& Spyrou, 2016; Zeng et al., 2016; Ramsahai et al., 2017; Morshed Osmani \& Rahman, 2018). These studies focus on ranking genes and discovering key driver genes in disease, and do not try to detect dysregulated pathways involved in disease. Other studies (Winter et al., 2012; Zeng et al., 2016) use PageRank to select topological 
159

160

161

162

163

164

165

166

167

168

169

170

171

172

173

174

175

176

177

178

179

180

181

182

183

184

185

186

187

188

189

190

191

192

193

194

195

196

197 important genes and simply see which pathways that these topological important genes are involved in. These PageRank-related approaches are very different from our approach.

Our approach embodied by PoTRA is motivated by the observation that the loss of connectivity is a common topological trait of cancer networks (Anglani et al., 2014), as well as the prior knowledge that a normal biological network includes a small number of well-connected hub nodes and a large number of nodes that are non-hubs (Albert, 2005; Khanin \& Wit, 2006; Zhu, Gerstein \& Snyder, 2007). However, from normal to cancer, the process of the network losing connectivity might be the process of disrupting the structure of the network, namely, the number of hub genes might be altered in cancer compared to that in normal or the distribution of topological ranks of genes might be altered. Thus, we hypothesize that if the number of hub genes is different in a pathway between normal and cancer, this pathway might be involved in cancer. Based on this hypothesis, we propose to detect pathways involved in cancer by testing if the number of hub genes for each pathway is different between normal and cancer samples.

Our approach embodied by PoTRA is also motivated by that the topological ranks of genes within biological pathways might be altered in cancer. Based on this, we propose to detect pathways involved in cancer by testing if the distribution of PageRank scores of genes for each pathway is altered between normal and cancer samples.

Therefore, the PoTRA approach computes topological ranks of genes in each pathway, and then detects pathways with significantly altered number of hub genes between normal and cancer, and, alternatively, detect pathways with significantly altered distributions of topological ranks of genes in corresponding pathways between two phenotypes. Namely, we first use the Google search PageRank algorithm to measure the relative topological ranks of genes in a biological pathway across different conditions. Then, we use Fisher's exact test to estimate if the number of hub genes in each pathway is significantly different between normal and cancer, and, alternatively, use the Kolmogorov-Smirnov test to estimate if the distribution of PageRank scores of genes in each pathway is significantly different between normal and cancer. As an illustration, we apply PoTRA to study hepatocellular carcinoma (HCC) and its subtypes and identify disease-relevant pathways. In conclusion, PoTRA is a new approach to explore and discover cancer-associated pathways. PoTRA can be used as a complement to other existing methods to enrich our understanding of the biological mechanisms behind cancer at the systemslevel.

\section{Materials and Methods}

Overview of the PoTRA method

Figure 1. Overview of the PoTRA method.

Below we detail the steps of the PoTRA method, as illustrated in Figure 1. 
198

199

200

201

202

203

204

205

206

207

208

209

210

211

212

213

214

215

216

217

218

219

220

221

222

223

224

225

226

227

228

229

230

231

232

233

234

235

To illustrate the PoTRA method, we use publicly available gene expression datasets from The Cancer Genome Atlas (TCGA) (https://cancergenome.nih.gov/) hepatocellular carcinoma (HCC) study. We analyze and contrast $50 \mathrm{HCC}$ samples and 50 tumor-adjacent normal samples ("normal samples" in future sections). In addition, the datasets also include gene expression profiles for several HCC subtypes. We further analyze and contrast 22 hepatitis B-induced HCC samples and 22 tumor-adjacent normal samples, 34 hepatitis C-induced HCC samples and 34 tumor-adjacent normal samples, and 50 alcohol-induced HCC samples and 50 tumor-adjacent normal samples. There are 20,531 gene expression values for each sample. Pathway information from the Kyoto Encyclopedia of Genes and Genomes (KEGG) database (Kanehisa \& Goto, 2000 ) is used. To date, there is much known about etiology of HCC (Beasley, 1988; Sanyal, Yoon \& Lencioni, 2010; Wang et al., 2012; Goossens \& Hoshida, 2015) and knowledge of pathways involved in HCC (Villanueva et al., 2008; Zhou et al., 2010; Wang et al., 2017), which makes it easier to illustrate and assess the PoTRA method.

\section{Construction of gene co-expression network for a pathway}

We apply the PoTRA method to gene expression profiles for several phenotypes, such as normal and cancer and cancer subtypes. First, we select genes for each pathway, using pathway information from KEGG. For each pathway, we determine the gene-gene interactions by using the Pearson's correlation to test each co-expressed gene pair. The test calculates a P-value for the association between each pair of genes. A significance threshold of 0.05 is used. When the Pvalue of a pair of genes is below 0.05 , we establish an edge between the corresponding two genes; otherwise, there is no edge between them. We implement it through a built-in function called "cor.test()" in the statistical software package R (https://www.r-project.org/). In this way, we can construct gene co-expression networks (i.e., pathways) for normal and cancer, separately. Of note, all the gene co-expression networks (i.e., pathways) used by PoTRA are undirected graphs, because co-expression networks only focus on gene pairs with a similar expression pattern across samples, in other words, the transcript levels of two co-expressed genes rise and fall together across samples.

In addition to construction of gene networks based on correlation alone, we, alternatively, also construct gene networks by combining the correlation with the pre-defined interaction from pathway databases.

3. PageRank analysis for genes within a pathway for normal and cancer

Based on the above constructed interactions within a pathway, we can observe that some genes are hub genes whereas others are non-hub genes. We apply the PageRank algorithm (Page et al., 1999) to obtain the corresponding topological importance for each gene within the pathway for normal and cancer, separately, see Figure 2. 
236 Figure 2. The topological rank analysis for each gene within a pathway. For genes within a

237

238

239

240

241

242

243

244

245

246

247

248

249

250

251

252

253

254

255

256

257

258

259

260

261

262

263

264

265

266

267

268

269

270

271

272

273

specified pathway, according to Step 2, we construct a corresponding gene co-expression network for normal and cancer, separately. Then we apply the PageRank method to obtain the topological importance of each gene for normal and cancer, separately. PR(gene i) normal represents the PageRank score of the gene i for normal samples, while PR(gene i) cancer represents the PageRank score of the gene i for cancer samples.

We implement it by using the page.rank() function from the igraph (Csárdi \& Nepusz, 2006) R package. As mentioned in Step 2, all the networks that we construct are undirected graphs. Thus, the PageRank algorithm used in our approach is based on undirected graphs.

\section{The PageRank algorithm}

The PageRank algorithm is used by the Google search engine to rank the importance of web pages, which is based on the assumption that the importance of a web page is high in a network if this web page has connections with other nodes of high importance. This idea is naturally applied to analyzing biological networks, where the importance of a gene is high if this gene is connected to other genes of high importance. In our study, the gene-gene network is an undirected graph where a node represents a gene and the edges can be defined by prior knowledge (e.g., KEGG database).

The output from the PageRank algorithm is a probability distribution representing the likelihood that a person randomly clicking on links will arrive at any particular web page. A probability is a numeric value between 0 and 1 . The sum of probabilities for all web pages is equal to 1 . The probability of a web page is proportional with the time spent at the web page when a person surfs the web. This idea can also be intuitively extended to ranking genes in gene networks where the probability of a gene is proportional with the time a research scientist spends looking and returning at the same gene when analyzing research results. For additional details of PageRank, please refer to (Page et al., 1999).

\section{Detect pathways with significant changes between normal and cancer scores}

4.1. Detect pathways with significantly altered number of hub genes between normal and cancer using Fisher's exact test

As mentioned above, PoTRA is motivated by the observation that the loss of connectivity is a common topological trait of cancer networks (Anglani et al., 2014) and the prior knowledge that a normal biological network includes a small number of well-connected hub nodes and a large number of nodes that are non-hubs (Albert, 2005; Khanin \& Wit, 2006; Zhu, Gerstein \& Snyder, 2007). From normal to cancer, the process of the network losing connectivity might be the process of disrupting the structure of the network, which can result in an altered number of hub genes between normal and cancer. Hence, a statistics that we compare between two phenotypes is the number of hub genes. The PageRank scores of all genes of a pathway form a distribution, 
274

275

276

277

278

279

280

281

282

283

284

285

286

287

288

289

290

291

292

293

294

295

296

297

298

299

300

301

302

303

304

305

306

307

308

and we use the $95^{\text {th }}$ percentile of the distribution (one-tail) in normal samples as cutoff value for hub genes for both normal and cancer samples. The genes in this pathway with PageRank scores that are above the cutoff value are identified as hub genes for this pathway. Then we count the number of hub genes for normal and cancer, separately. Next, we use Fisher's exact test to assess if the number of hub genes is significantly different between normal and cancer. For details, see Table 1.

Table 1. Contingency table for Fisher's exact test. We use the $95^{\text {th }}$ percentile of the distribution (one-tail) in normal samples as cutoff value for hub genes for both normal and cancer samples. The value "a" represents the number of genes whose PageRank scores are below the cutoff value for normal samples. The value " $b$ " represents the number of genes whose PageRank scores are above the cutoff value for normal samples. The values "c" and " $d$ " are the corresponding values for cancer. We use Fisher's exact test to assess if the number of hub genes is significantly different between normal and cancer.

Fisher's exact test estimates the probability of obtaining any such set of values, given by the hypergeometric distribution:

$$
\mathbf{P}=\frac{\left(\begin{array}{c}
a+b \\
a
\end{array}\right)\left(\begin{array}{c}
c+d \\
c
\end{array}\right)}{\left(\begin{array}{c}
n \\
a+c
\end{array}\right)}=\frac{(a+b) !(c+d) !(a+c) !(b+d) !}{a ! b ! c ! n !}
$$

where $\mathrm{n}=\mathrm{a}+\mathrm{b}+\mathrm{c}+\mathrm{d}$, and $\left(\begin{array}{l}i \\ j\end{array}\right)$ is the binomial coefficient and the symbol "'?" indicates the factorial operator.

Formula 1 gives the exact hypergeometric probability of observing this particular arrangement of the data, assuming the given marginal totals, on the null hypothesis that the number of hub genes is the same for a specified pathway between normal and cancer. If this test statistic is significant, it indicates that there is a significantly different number of hub genes between normal and cancer, thereby this pathway might be involved in cancer. By studying many pathways from the KEGG database we generate a multiple hypothesis testing problem. We address this issue by correcting the P-values calculated for each pathway using the False Discovery Rate (FDR) approach, using the Benjamini and Hochberg procedure (Benjamini \& Hochberg, 1995).

\subsection{Detect pathways with significantly altered distributions of PageRank scores for genes} between normal and cancer using Kolmogorov-Smirnov test

Alternatively, PoTRA is implemented by testing if the distribution of PageRank scores of genes is altered between normal and cancer, using the two-sample Kolmogorov-Smirnov test. The twosample Kolmogorov-Smirnov test is a nonparametric test of equality of continuous, onedimensional probability distributions that can be used to compare two samples. The 
309

310

311

312

313

314

315

316

317

318

319

320

321

322

323

324

325

326

327

328

329

330

331

\section{2}

333

334

335

336

337

338

339

340

341

Kolmogorov-Smirnov statistic quantifies a distance between the empirical distribution functions of two samples. The null distribution of this statistic is calculated under the null hypothesis that the samples are drawn from the same distribution.

The empirical distribution function $F_{n}$ for $n$ i.i.d. observation $X_{i}$ is defined as:

$$
\mathrm{F}_{\mathrm{n}}(\mathrm{x})=\frac{1}{n} \sum_{i=1}^{n} I_{|-\infty, \mathrm{x}|}\left(\mathrm{X}_{\mathrm{i}}\right)
$$

where $\mathrm{I}_{\mid-\infty, \mathrm{x}}\left(\mathrm{X}_{\mathrm{i}}\right)$ is the indicator function, equal to 1 if $\mathrm{X}_{\mathrm{i}} \leq \mathrm{x}$ and equal to 0 otherwise.

The two-sample Kolmogorov-Smirnov test may be used to test if two underlying onedimensional probability distributions differ. In this case, the Kolmogorov-Smirnov statistic is:

$$
\mathrm{D}_{\mathrm{n}, \mathrm{m}}=\operatorname{Sup}_{x}\left|F_{1, \mathrm{n}}(\mathrm{x})-\mathrm{F}_{2, \mathrm{~m}}(\mathrm{x})\right|
$$

where $F_{1, \mathrm{n}}$ and $\mathrm{F}_{2, \mathrm{~m}}$ are the empirical distribution functions of the first and the second sample respectively, and sup is the supremum function.

If the test statistic for a specific pathway is significant, it indicates that the two underlying distributions differ between normal and cancer, thereby this pathway is involved in cancer. By studying many pathways from the KEGG database we generate a multiple hypothesis testing problem. We address this issue by correcting the P-values calculated for each pathway using the False Discovery Rate (FDR) approach, using the Benjamini and Hochberg procedure (Benjamini \& Hochberg, 1995).

\section{Software tools}

All the analysis is conducted using the $\mathrm{R}$ statistical programming language, using the following R Biocoductor packages: graphite for pathway databases, igraph for PageRank function and graph for visualization.

\section{Results}

We apply PoTRA to analyze and contrast 50 HCC samples and 50 tumor-adjacent normal samples. All data come from The Cancer Genome Atlas (TCGA) hepatocellular carcinoma (HCC) study.

1. PoTRA for HCC vs. normal samples using correlation-based networks

To illustrate the PoTRA method, we use a cancer-associated pathway, "MAPK signaling pathway", as an example in the following section.

1.1. Construction of a gene co-expression network for a pathway 
342

343

344

345

346

347

348

349

350

351

352

353

354

355

356

357

358

359

360

361

362

363

364

365

366

367

368

369

370

371

372

373

374

375

376

377

378

379

As suggested before, "MAPK signaling pathway" might be comprised of different interactions between genes under different conditions, such as normal versus cancer conditions. First, we need to find the genes that this pathway consists of by using the KEGG database. In practice, we implement it by using an R package called graphite. Second, for the genes of this pathway, we identify the interactions between genes for normal and cancer samples, separately. There are 14,005 edges for normal and 5,170 edges for cancer. For the information of "MAPK signaling pathway" in normal and cancer samples, see Supplementary Table S1.

\subsection{PageRank analysis for genes within a pathway for normal and cancer}

Based on the interactions identified in the previous section, we can obtain a PageRank score for each gene in "MAPK signaling pathway" for normal and cancer, separately, which quantifies the influence of a gene on the activity of other genes in this pathway. For the results for this step, 2 vectors with 250 PageRank values, one for normal and one for cancer, separately, see Supplementary Table S1. As previously mentioned, the PageRank values in each vector add up to 1 .

Figure 3 illustrates the distributions of PageRank scores for genes in "MAPK signaling pathway" for normal and cancer, separately.

\section{Figure 3. The kernel density distribution of PageRank scores of genes in "MAPK signaling} pathway". The red line shows the kernel density distribution of PageRank scores for cancer and the black one is for normal samples. Note that the mean for the two distributions is the same, i.e., mean $=1 / \mathrm{N}=0.004$, where $\mathrm{N}=250$ is the number of genes in the "MAPK signaling pathway" pathway. We use the 95 th-percentile cutoff $(=0.006035)$ of the kernel distribution in normal samples as cutoff for hub genes for both normal and cancer samples.

The number of hub genes changes. While there are only 13, strongly-connected (with more edges, an average of 121 edges) hub genes in the normal samples, there are 40 hub genes in the cancer samples, more loosely-connected (with fewer edges, an average of 75 edges).

As mentioned before, the process of the network losing connectivity might be the process of disrupting the structure of the network which includes a small number of hub nodes and a large number of non-hub nodes, namely, the number of hub genes might be altered in cancer compared to that in normal. The altered number of hub genes might be a trait of a pathway in cancer, which suggests the pathway is involved in cancer if the change in hub gene number is statistically significant.

Importantly, we find increased variance (median change 5.2 -fold for the MAPK pathway, or 6.1 fold change for all genes) of gene expression in cancer samples compared to normal samples, which is included in Supplementary Table S1. The increased variance in cancer samples results in lower Pearson correlations between genes in cancer, which leads to lower correlations and loss of connectivity in cancer. This loss of connectivity can lead to the disruption of the structure of 
380

381

382

383

384

385

386

387

388

389

390

391

392

393

394

395

396

397

398

399

400

401

402

403

404

405

406

407

408

409

410

411

412

413

414

415

416

the biological networks. To quantify these changes in the network topology between normal and disease, we use Fisher's exact test to identify changes in the number of network hub genes, and the Kolmogorov Smirnov test to test if the distributions of PageRank scores are significantly different between normal and cancer. These tests are described below.

\subsubsection{Fisher's exact test for comparing the number of hub genes in the pathway}

We next use Fisher's exact test to test if the number of hub genes for "MAPK signaling pathway" is significantly different between normal and cancer. The result for the "MAPK signaling pathway" pathway is included in Table 2.

\section{Table 2. The "MAPK signaling pathway" pathway identified by PoTRA for HCC using} Fisher's exact test. The P value is adjusted by False Discovery Rate (FDR).

The low P-value in Table 2 indicates that the number of hub genes in cancer samples is significantly different from that in normal samples, suggesting that the "MAPK signaling pathway" pathway is involved in HCC. This example suggests that a normal biological network includes a small number of hub nodes and a large number of non-hub nodes. Moreover, the loss of connectivity from normal to cancer might lead to disrupting the structure of the network in cancer, thereby resulting in the fact that the number of hub genes is altered in cancer compared to that in normal.

Then we apply the same approach to other pathways from KEGG to compare HCC vs. normal samples. The significant pathways are shown in Table 3.

\section{Table 3. The significant KEGG pathways identified by PoTRA for HCC using Fisher's exact test. FDR adjusted P-values are below 0.05 .}

We find three significant pathways with altered number of hub genes between normal and cancer. It is well known that these three pathways are strongly associated with cancer in general. MAPK signaling pathway plays a role in the regulation of gene expression, cellular growth, and survival (Knight \& Irving, 2014). Abnormal MAPK signaling might lead to uncontrolled or increased cell proliferation and resistance to apoptosis (Santarpia, Lippman \& El-Naggar, 2012; Burotto et al., 2014). Interestingly, we also find that loss of connectivity and the larger number of hub genes for cancer are characteristics of the other two pathways as well.

The levels for parameter thresholds can be varied. Hence, we also have tried different threshold levels for hub genes in Fisher's exact test: 0.9, 0.85, 0.8, 0.75 and 0.7. For these threshold levels, the p-values are larger than that when the threshold is 0.95 , and we do not find any significant pathways using Fisher's exact test. This suggests that, for this test, using a limited number of genes of higher "hubness" (higher PageRank values) is important when comparing normal and cancer. 
418

1.3.2. The Kolmogorov-Smirnov test for comparing distributions of PageRank scores of genes for pathways

We next use the Kolmogorov-Smirnov test to test if the two distributions of PageRank scores of genes for "MAPK signaling pathway" are significantly different between normal and cancer. The result for the "MAPK signaling pathway" pathway is included in Table 4.

\section{Table 4. The "MAPK signaling pathway" pathway identified by PoTRA for HCC using the} Kolmogorov-Smirnov test. The P value is adjusted by False Discovery Rate (FDR).

The low P-value in Table 4 indicates that the distribution of PageRank scores of genes in cancer samples is significantly different from that in normal samples, suggesting that the "MAPK signaling pathway" pathway is involved in HCC. MAPK signaling pathway plays a role in the regulation of gene expression, cellular growth, and survival (Knight \& Irving, 2014). Abnormal MAPK signaling might lead to uncontrolled or increased cell proliferation and resistance to apoptosis (Santarpia, Lippman \& El-Naggar, 2012; Burotto et al., 2014).

Then we apply the same approach to other pathways from KEGG to compare HCC with normal samples. The significant pathways are shown in Table 5.

\section{Table 5. The significant KEGG pathways identified by PoTRA for HCC using the Kolmogorov-Smirnov test. FDR adjusted P-values are below 0.05.}

We find three significant pathways with altered distribution of PageRank scores of genes between normal and cancer. In addition to "MAPK signaling pathway", we find two other pathways: "RNA transport" and "mRNA surveillance pathway". For the "RNA transport" pathway, it is well known that the nuclear export of mRNA is intrinsically linked to the cotranscriptional processing of nascent transcripts synthesized by the RNA polymerase II. This functional coupling is very important for the survival of cells allowing for nuclear export of fully processed transcripts, which could lead to genome instability and to various forms of cancer (Siddiqui \& Borden, 2012; Hautbergue, 2017). The "mRNA surveillance pathway" is a quality control mechanism that detects and degrades abnormal mRNAs, which includes nonsensemediated mRNA decay (NMD), nonstop mRNA decay (NSD), and no-go decay (NGD). NMD can degrade PTC (Premature termination codons)-containing transcripts which cause a large proportion of human cancers (Lindeboom, Supek \& Lehner, 2016). Nonstop decay is the mechanism of identifying and disposing aberrant transcripts that lack in-frame stop codons. These transcripts are identified during translation when the ribosome arrives at the 3' end of the mRNA and stalls at the end of the poly(A) tail. This surveillance mechanism protects the cells from potentially harmful truncated proteins, but it may also be involved in mediating critical cellular functions of transcripts that are prone to stop codon read-through, which have implications in human cancers (Klauer \& van Hoof, 2012). No-go decay (NGD) is a eukaryotic 
455

456

457

458

459

460

461

462

463

464

465

466

467

468

469

470

471

472

473

474

475

476

477

478

479

480

481

482

483

484

485

486

487

488

489

490

491

492

quality control mechanism that evolved to cope with translational arrests (Simms, Yan \& Zaher, 2017). These above processes are strongly related to cancer.

\subsection{PoTRA for cancer subtype analysis}

1.4.1. Fisher's exact test for cancer subtype analysis

Many complex diseases have subtypes and/or can be classified into different categories based on diagnosis, pathology, phenotype characteristics, etc. To further assess the PoTRA method, we apply it to several subtypes of the HCC TCGA data. There are several risk factors associated with HCC, such as hepatitis B, hepatitis C and alcohol (Beasley, 1988; Sanyal, Yoon \& Lencioni, 2010; Hoshida et al., 2014; Goossens \& Hoshida, 2015). Here, we apply PoTRA to compare these three subtypes of HCC samples with normal samples.

Table 6 illustrates the Fisher's exact test results for comparing normal with hepatitis B-induced HCC samples.

\section{Table 6. The significant KEGG pathways identified by PoTRA for hepatitis B-induced HCC using Fisher's exact test. FDR adjusted P-values are below 0.05.}

There is one common pathway, Pathways in cancer, between Table 6 and Table 3. There are seven other new pathways, which are very interesting and associated with the hepatitis Binduced HCC. First, the "Hepatitis B" pathway is detected by our method. Hepatitis B is the most important and direct factor causing hepatitis B-induced HCC. In addition, we find two other pathways, HTLV-I (Human T-cell leukemia virus type I) infection and Epstein-Barr virus infection, which are strongly associated with virus infection and cancer. This is consistent with the viral pathology of hepatitis B-induced HCC. Besides, some studies show that hepatitis B virus infection can contribute to the impairment of insulin signaling, which is another pathway identified by PoTRA (Kim, Kim \& Cheong, 2010; Barthel et al., 2016). Finally, the other three pathways, Hippo signaling pathway, Neurotrophin signaling pathway and mTOR signaling pathway are associated with cancer in general. Hippo signaling pathway is reported to be able to control organ size through regulating cell proliferation and apoptosis (Saucedo \& Edgar, 2007; Pan, 2010). It is reported that neurotrophins can regulate cancer stem cells (Chopin et al., 2016), and neurotrophins contribute to pro-survival signaling in many different types of cancer (Molloy, Read \& Gorman, 2011). mTOR signaling pathway is a well-known cancer-associated pathway. Alterations of mTOR signaling pathway have significant effects on cancer progression. The major components of mTOR signaling pathway are critical effectors in cell signaling pathways commonly deregulated in cancers (Guertin \& Sabatini, 2007; Villanueva et al., 2008; Pópulo, Lopes \& Soares, 2012).

These results suggest that PoTRA can be used to identify not only the pathways associated with cancer in general, but also those pathways associated with cancer subtypes, such as hepatitis Binduced HCC specifically. 
493

494

495

496

497

498

499

500

501

502

503

504

505

506

507

508

509

510

511

512

513

514

515

516

517

518

519

520

521

522

523

524

525

526

527

528

529

530

531

Results of the PoTRA analysis from two other HCC subtypes, hepatitis C-induced HCC and alcohol-induced HCC, are included in Table 7 and Table 8, respectively.

\section{Table 7. The significant KEGG pathways identified by PoTRA for hepatitis C-induced} HCC using Fisher's exact test. FDR adjusted P-values are below 0.05.

In Table 5, we find two common pathways, Pathways in cancer and MAPK signaling pathway, between Table 5 and Table 3. Among the other pathways, we find several pathways related to cancer generally and hepatitis C-induced HCC specifically. First, HTLV-I infection is also listed in this table, and, as mentioned above, is associated with virus infection and cancer. Almost all other pathways are associated with cancer in general. PI3K-Akt signaling pathway is a key regulator of normal cellular processes involved in cell growth, proliferation, motility, survival, and apoptosis (Porta, Paglino \& Mosca, 2014). The Proteoglycans in cancer pathway is involved in regulation of proteoglycans, heavily glycosylated proteins present especially in connective tissue in cancer. Rap1 signaling pathway is reported to be involved in cancer cell migration, invasion and metastasis (Bailey, 2009; Zhang et al., 2017). The cAMP signaling pathway regulates a number of biological processes, such as cell growth and adhesion, neuronal signaling, energy homeostasis and muscle relaxation (Fajardo, Piazza \& Tinsley, 2014). The key component of Focal adhesion pathway, Focal adhesion kinase (FAK), is reported to enable activation by growth factor receptors or integrins in different types of cancers. FAK is an important mediator of cell proliferation, cell migration, cell growth (Golubovskaya, Kweh \& Cance, 2009; Tai, Chen \& Shen, 2015). A large volume of literature shows Ras signaling pathway is involved in several aspects of normal cell growth and malignant transformation, and plays an important role in cancer development and progression (Vojtek \& Der, 1998; Downward, 2003; Santarpia, Lippman \& El-Naggar, 2012; Knight \& Irving, 2014). FoxO signaling pathway is involved in the regulation of the cell cycle, apoptosis and metabolism (Schmidt et al., 2002; Fu \& Tindall, 2008; Gross, van den Heuvel \& Birnbaum, 2008). Besides, activity of FoxO signaling pathway also affects stem cell maintenance and lifespan (Eijkelenboom \& Burgering, 2013). ErbB signaling pathway plays roles in cancer development and progression (Hynes \& Lane, 2005; Seshacharyulu et al., 2012), as well as in cancer cell migration and invasion (AppertCollin et al., 2015). ErbB signaling pathway is associated with the development of a wide variety of types of solid tumor if ErbB signaling is excessive (Cho \& Leahy, 2002). The Axon guidance pathway is also reported to regulate cell migration and apoptosis, and be associated with tumorigenesis (Chédotal, Kerjan \& Moreau-Fauvarque, 2005).

\section{Table 8. The significant KEGG pathways identified by PoTRA for alcohol-induced HCC using Fisher's exact test. FDR adjusted P-values are below 0.05.}

We find two common pathways between Table 8 and Table 3, MAPK signaling pathway and Pathways in cancer. As mentioned above, PI3K-Akt signaling pathway also plays an important role in cancer (Porta, Paglino \& Mosca, 2014). 
533 1.4.2. Kolmogorov-Smirnov test for cancer subtype analysis

534 Table 9 illustrates the Kolmogorov-Smirnov test results for comparing normal with hepatitis C-

535 induced HCC samples.

536

Table 9. The significant KEGG pathways identified by PoTRA for hepatitis $C$-induced HCC using the Kolmogorov-Smirnov test. FDR adjusted P-values are below 0.05.

539

540

541

542

543

544

545

546

547

548

549

550

551

552

553

554

555

556

557

558

559

560

561

562

563

564

565

566

567

568

In Table 9, we find two common pathways, RNA transport and MAPK signaling pathway, between Table 9 and Table 5. Among the other pathways, we find pathways related to cancer generally and hepatitis C-induced HCC specifically. First, HTLV-I infection is also listed in this table, and, as mentioned above, is associated with virus infection and cancer. PI3K-Akt signaling pathway is a key regulator of normal cellular processes involved in cell growth, proliferation, motility, survival, and apoptosis (Porta, Paglino \& Mosca, 2014). Importantly, hepatitis C virus activates PI3K-Akt signaling to enhance entry and replication, and meanwhile PI3K-Akt signaling pathway also can increase HCV translation (Liu et al., 2012; Shi, Hoffman \& Liu, 2016), which suggest that PI3K-Akt signaling pathway is associated with hepatitis $C$ virus infection specifically. The "Pathways in cancer" pathway is associated with cancer in general. The "Proteoglycans in cancer" pathway is involved in regulation of proteoglycans, heavily glycosylated proteins present especially in connective tissue in cancer (Iozzo \& Sanderson, 2011; Baghy et al., 2016).

Results of the PoTRA analysis from two other HCC subtypes, alcohol-induced HCC and hepatitis B-induced HCC, are included in Table 10 and Table 11, respectively.

\section{Table 10. The significant KEGG pathways identified by PoTRA for alcohol-induced HCC} using the Kolmogorov-Smirnov test. FDR adjusted P-values are below 0.05.

We find three common pathways between Table 10 and Table 5, RNA transport, MAPK signaling pathway and mRNA surveillance pathway. As mentioned above, the "Pathways in cancer" pathway is strongly associated with cancer in general and PI3K-Akt signaling pathway also plays an important role in cancer (Porta, Paglino \& Mosca, 2014).

\section{Table 11. The significant KEGG pathways identified by PoTRA for hepatitis B-induced}

\section{HCC using the Kolmogorov-Smirnov test. FDR adjusted P-values are below 0.05.}

There is no common pathway between Table 11 and Table 5. Very interestingly, for the association between hepatitis B-induced HCC and the "Primary bile acid biosynthesis" pathway, some studies demonstrate that hepatitis B virus infection can alter bile acid metabolism as a consequence of impaired bile acid uptake (Oehler et al., 2014; Geier, 2014). Also, some studies show that hepatitis B virus infection can contribute to the impairment of insulin signaling.(Kim, 
569 Kim \& Cheong, 2010; Barthel et al., 2016). These two pathways mentioned are specifically 570 related to hepatitis B virus infection. Arginine and proline metabolism is one of the central 571 pathways for the biosynthesis of the amino acids arginine and proline from glutamate. Some 572 studies have suggested that altered arginine and proline metabolism is linked to metastasis 573 formation in cancer (Elia et al., 2017). Arginine serves as an intermediate in the urea cycle and as 574 a precursor for protein, polyamine, creatine and nitric oxide (NO) biosynthesis. NO may 575 influence tumor initiation, promotion, and progression, tumor-cell adhesion, apoptosis

576 angiogenesis, differentiation, chemosensitivity, radiosensitivity, and tumor-induced 577 immunosuppression (Lind, 2004).

578

2. PoTRA for HCC vs. normal samples using networks that combine correlation-based networks with curated interaction networks

581

582

583

584

585

586

587

588

589

590

591

592

593

594

595

596

597

598

599

600

601

602

603

604

The above results show that the Fisher's exact test and the Kolmogorov-Smirnov test can identify cancer-related pathways based on correlation networks. In addition to correlation networks, in this section we apply PoTRA to gene networks constructed by intersecting correlation networks with pre-defined networks from the KEGG database. As performed in the previous section we investigate if Fisher's exact test and Kolmogorov-Smirnov test are still able to robustly discover cancer-associated pathways and differential pathways between HCC and subtypes of HCC based on the combined networks.

\subsection{Fisher's exact test for HCC and subtypes of HCC}

For HCC, we use Fisher's exact test and identify no significant pathway with altered number of hub genes between normal and HCC.

For hepatitis C-induced HCC, we identify two significant pathways, listed in Table 12.

\section{Table 12. The significant KEGG pathways identified by PoTRA for hepatitis C-induced} HCC using the Fisher's exact test based on combined networks. FDR adjusted P-values are below 0.05 . E.comb.normal represents the number of edges in the combined network for normal samples, while E.comb.case is for cancer samples, respectively.

As mentioned above, Epstein-Barr virus infection is a pathway associated with virus infection, which is specifically related to hepatitis C induced HCC. P53 signaling pathway is a classical oncogenic pathway, and it can regulate the cell cycle, apoptosis and help prevent cancer. The major component of p53 signaling pathway, p53 protein, is most frequently altered in human cancer (May \& May, 1999; Sherr \& McCormick, 2002; Sui et al., 2011; Stegh, 2012).

For hepatitis B-induced HCC, we identify one significant pathway, listed in Table 13. 
605

606

607

608

609

610

611

612

613

614

615

616

617

618

619

620

621

622

623

624

625

626

627

628

629

630

631

632

633

634

635

636

637

638

639

640

641

642

643

Table 13. The significant KEGG pathways identified by PoTRA for hepatitis B-induced HCC using the Fisher's exact test based on combined networks. FDR adjusted P-values are below 0.05. E.comb.normal represents the number of edges in the combined network for normal samples, while E.comb.case is for cancer samples, respectively.

As such, we identify the same significant pathway "Epstein-Barr virus infection", which is also specific for hepatitis $\mathrm{C}$-induced $\mathrm{HCC}$, discussed above.

For alcohol-induced HCC, we identify no significant pathways.

First, we can observe a large loss of connectivity for combined networks vs. correlation networks in normal and in cancer. For example, Table 13 lists only 84 edges in normal and 22 edges in cancer samples for the Epstein-Barr virus infection pathway using combined correlation and curated interactions. This is in contrast to 1524 edges in normal and 435 edges in cancer shown in Table 6 for the same pathway, when using all the correlation-based edges. We can observe that the proportion of edges in normal to cancer for the correlation network and the combined network are approximately the same (about 4:1). On average, we observe a reduction by, a factor of 23.18 in the number of edges between correlation and combined networks. However, the PoTRA-Fisher's exact test still can identify pathways specifically involved in subtypes of HCC.

Furthermore, because combined networks have a large loss of connectivity from correlation networks to combined networks, the PageRank scores of genes are more evenly distributed in combined networks than in correlation networks. Hence, fewer significant pathways with altered number of hub genes between normal and cancer are identified for combined networks than that for correlation networks. Although Fisher's exact test identifies fewer significant pathways, this test can still discover differential pathways specific for subtypes of HCC between normal and cancer, suggesting that the Fisher's exact test is able to robustly discover pathways involved in cancer and subtypes of cancer.

\subsection{Kolmogorov-Smirnov test for HCC and subtypes of HCC}

All significant pathways for HCC and each subtype of HCC (hepatitis C-induced HCC, hepatitis B-induced HCC and alcohol-induced HCC) using the Kolmogorov-Smirnov test on combined networks are included in Supplementary Table S2, Supplementary Table S3, Supplementary Table S4 and Supplementary Table S5, respectively. Here, we review the top 10 significant pathways from HCC and each subtype of HCC.

Among the results of the PoTRA analysis for HCC in Supplementary Table S2, we can find many pathways involved in HCC in the top 10 significant pathways, such as Cytokine-cytokine receptor interaction, cAMP signaling pathway, p53 signaling pathway, etc. Cytokine-cytokine receptor interaction exerts a vast array of immunoregulatory actions critical to cancers (Schreiber \& Walter, 2010; Spangler et al., 2015). The cAMP signaling pathway regulates a number of biological processes, such as cell growth and adhesion, neuronal signaling, energy homeostasis and muscle relaxation (Fajardo, Piazza \& Tinsley, 2014). P53 signaling pathway is a classical oncogenic pathway as mentioned above. 
644 Among the results for hepatitis C-induced HCC (Supplementary Table S3), we find seven non-

645

646

647

648

649

650

651

652

653

654

655

656

657

658

659

660

661

662

663

664

665

666

667

668

669

670

671

672

673

\section{4}

675

676

677

678

679

680

681

682

common pathways for this subtype of HCC in the top 10 pathways, such as MAPK signaling pathway, Calcium signaling pathway, cGMP-PKG signaling pathway, Adrenergic signaling in cardiomyocytes, HIF-1 signaling pathway, Toll-like receptor signaling pathway and Insulin resistance. Among them, some of them are strongly related to immune system and inflammation, such as cGMP-PKG signaling pathway, HIF-1 signaling pathway, Toll-like receptor signaling pathway and Insulin resistance. These immune- and inflammation-related pathways are also specific for hepatitis $\mathrm{C}$ (virus infection)-induced HCC.

Among the results for hepatitis B-induced HCC (Supplementary Table S4), we also find seven non-common pathways between this subtype of HCC and HCC in the top 10 pathways, such as Adrenergic signaling in cardiomyocytes, Breast cancer, Calcium signaling pathway, cGMP-PKG signaling pathway, MAPK signaling pathway, PI3K-Akt signaling pathway and Tuberculosis. Among them, there are some pathways related to immune system and inflammation, such as cGMP-PKG signaling pathway and PI3K-Akt signaling pathway, which are specific for hepatitis $\mathrm{B}$ (virus infection)-induced HCC.

Among the results for alcohol-induced HCC (Supplementary Table S5), we also find four noncommon pathways between this subtype of HCC and HCC in the top 10 pathways, such as MAPK signaling pathway, Pathways in cancer, Calcium signaling pathway and Progesteronemediated oocyte maturation. Among these four pathways, Calcium signaling pathway is shown to be specifically associated with alcohol in some studies (Gruol \& Parsons, 1996; Li, Li \& Guo, 2014; Bartlett et al., 2017).

As mentioned above, the combined networks, which intersect correlation networks with curated database networks, have a large loss of gene-gene interactions when compared to correlation networks. Hence, the distribution of PageRank scores of genes might be changed more from normal to cancer than that in correlation networks. Hence, more significant pathways with altered distribution of PageRank scores of genes are identified for combined networks than that for correlation networks. Although the Kolmogorov-Smirnov test identifies a large amount of significant pathways, we can use this test for combined networks to rank the pathways associated with cancer.

\section{Discussion}

We propose a PageRank-based method, Pathway of Topological Rank Analysis (PoTRA), for identifying pathways involved in cancer. PoTRA is motivated by the observation that the loss of connectivity is a common topological trait of cancer networks (Anglani et al., 2014) and the prior knowledge that a normal biological network includes a small number of hub nodes and a large number of non-hub nodes (Albert, 2005; Khanin \& Wit, 2006; Zhu, Gerstein \& Snyder, 2007). From normal to cancer, the process of the network losing connectivity might be the process of disrupting the structure of the network, which can result in an altered number of hub genes between normal and cancer. The PoTRA analysis is based on topological ranks of genes in 
683 biological pathways, and PoTRA detects pathways involved in cancer by testing if the number of 684 hub genes in pathways is altered between normal and cancer.

685 To illustrate the method, PoTRA is applied to several TCGA hepatocellular carcinoma datasets. 686 The results in our study are in agreement with prior knowledge of HCC from literature. We find 687 that a high proportion of statistically significant pathways play important roles in cancer, 688 indicating that the altered number of hub genes for these pathways might indeed be a reflection 689 of the underlying biological causes that lead to cancer. Moreover, in the comparison between 690 normal and each subtype of HCC, most importantly, the "Hepatitis B" pathway and several 691 pathways associated with virus infection dramatically become significant pathways in hepatitis

692 B-induced HCC, suggesting that PoTRA is capable of detecting pathways associated with 693 disease subtypes. We also find several pathways associated with HCC generally and subtype 694 specifically in hepatitis C-induced HCC and in alcohol-induced HCC.

695 In our approach, the correlation method is used to construct gene co-expression networks for 696 normal and cancer, respectively. A gene co-expression network is an undirected graph, where 697 each node represents a gene, and each edge is established if there is a significant co-expression 698 relationship between two genes (Stuart et al., 2003). Stuart JM, et al. (Stuart et al., 2003) found 69922,163 co-expression relationships, each of which has been conserved across evolution, 700 suggesting that the co-expressions between genes confers a selective advantage and thus these genes are functionally related. Gene co-expression networks are biologically interesting since coexpressed genes might be controlled by members of the same pathway, or the same transcriptional regulatory program or protein complex (Weirauch, 2011), and could be functionally related, suggesting that co-expression is common in the human genome. A gene coexpression network can be constructed by looking for pairs of genes with a similar expression pattern across samples, i.e., the transcript levels of two co-expressed genes rise and fall together across samples. As method of network construction, we use Pearson's correlation in consideration of saving computing time, because the other methods are relatively computationally intensive. In addition, we also use combined networks by taking the intersection of KEGG curated networks and correlation networks, which increases the reliability of network construction. This approach also shows that the results using correlation networks and the results using combined networks are consistent.

Here, we construct gene networks using two ways. One approach is based on correlation networks, while the other approach is to combine (intersect) the correlation networks with predefined networks from pathway databases. Because the combined networks lose a large amount of gene-gene interactions from the correlation networks, the power is reduced and the PageRank scores of genes tend to more evenly distributed in combined networks than that in correlation networks. Hence, fewer significant pathways with altered number of hub genes between two phenotypes are identified for combined networks than that for correlation networks. Moreover, the distribution of topological ranks of genes might be changed more from normal to cancer than that in correlation networks. Thus, more significant pathways with altered distribution of PageRank scores of genes are identified for combined networks than that for correlation 
723

724

725

726

727

728

729

730

731

732

733

734

735

736

737

738

739

740

741

742

743

744

745

746

747

748

749

750

751

752

753

754

755

756

networks. Although the Kolmogorov-Smirnov test identifies a number of significant pathways for combined networks, we can use the Kolmogorov-Smirnov test to rank those pathways.

We apply the Fisher's exact test and the Kolmogorov-Smirnov test to analyze the PageRank scores for the two types of gene networks as mentioned above. The corresponding results suggest that the Fisher's exact test and the Kolmogorov-Smirnov test can identify and rank cancerassociated pathways. This suggests that the PoTRA method is robust to choice of network building approach and to statistical analysis method for identification of cancer-related pathways.

\section{Future Directions}

The hypothesis of our study is based on the fact that the loss of connectivity is a common topological trait of cancer networks (Anglani et al., 2014). It is not yet well understood if this trait is a characteristic of other complex diseases. Thus, we need to be cautious about the applicability of this method to other diseases. However, this trait could be applicable to other complex diseases. Thus, although PoTRA is motivated by work on cancer, it could apply to other complex diseases as well. This area needs to be further investigated.

In this study, we apply PoTRA to pre-defined biological pathways, from the well-curated KEGG pathway database. However, the PoTRA method can also be applied to any set of genes of interest, such as functional gene subnetworks. This could be an interesting area to further explore.

In this article, we focus on the details of the PoTRA methodology. For different pathway databases, such as Reactome, Biocarta, etc., the method would apply in a similar manner, while the final results might vary slightly, depending on the data being used. This direction - changes in analysis results based on different pathway databases - would be an interesting area to investigate in the future.

\section{Conclusion}

In summary, PoTRA provides a new method for detecting cancer-associated pathways. PoTRA may be used to augment existing methods and provide a richer, more systematic understanding of cancer mechanisms.

\section{Availability}

R software to carry out the PoTRA computation is available via http://www.dinulab.org/tools.

\section{Reference}


757 Albert R. 2005. Scale-free networks in cell biology. Journal of Cell Science 118:4947-4957. DOI:

$758 \quad$ 10.1242/jcs.02714.

759 Anglani R., Creanza TM., Liuzzi VC., Piepoli A., Panza A., Andriulli A., Ancona N. 2014. Loss of Connectivity

760 in Cancer Co-Expression Networks. PLOS ONE 9:e87075. DOI: 10.1371/journal.pone.0087075.

761 Appert-Collin A., Hubert P., Crémel G., Bennasroune A. 2015. Role of ErbB Receptors in Cancer Cell

762 Migration and Invasion. Frontiers in Pharmacology 6. DOI: 10.3389/fphar.2015.00283.

763 Baghy K., Tátrai P., Regős E., Kovalszky I. 2016. Proteoglycans in liver cancer. World Journal of

764 Gastroenterology 22:379-393. DOI: 10.3748/wjg.v22.i1.379.

765

Bailey CL. 2009. The Roles of Rap1 in Cancer Metastasis and Pancreatic Islet Beta Cell Function.

766 Barthel SR., Medvedev R., Heinrich T., Büchner SM., Kettern N., Hildt E. 2016. Hepatitis B virus inhibits insulin receptor signaling and impairs liver regeneration via intracellular retention of the insulin Journal of Physiology 595:3143-3164. DOI: 10.1113/JP273891.

Beasley RP. 1988. Hepatitis B virus. The major etiology of hepatocellular carcinoma. Cancer 61:19421956. conserved gene-gene relationships derived from gene co-expression network analysis on breast cancer data. Scientific Reports 6:20518. DOI: 10.1038/srep20518. 
781 Burotto M., Chiou VL., Lee J-M., Kohn EC. 2014. The MAPK pathway across different malignancies: A new 782 perspective. Cancer 120:3446-3456. DOI: 10.1002/cncr.28864.

783 Chédotal A., Kerjan G., Moreau-Fauvarque C. 2005. The brain within the tumor: new roles for axon guidance molecules in cancers. Cell Death and Differentiation 12:1044-1056. DOI: 10.1038/sj.cdd.4401707.

786

787

788

789

790

791

792

793

794

795

796

797

798

799

800

801

802

803

Cho H-S., Leahy DJ. 2002. Structure of the extracellular region of HER3 reveals an interdomain tether. Science (New York, N.Y.) 297:1330-1333. DOI: 10.1126/science.1074611.

Choi Y., Kendziorski C. 2009. Statistical methods for gene set co-expression analysis. Bioinformatics (Oxford, England) 25:2780-2786. DOI: 10.1093/bioinformatics/btp502.

Chopin V., Lagadec C., Toillon R-A., Le Bourhis X. 2016. Neurotrophin signaling in cancer stem cells. Cellular and molecular life sciences: CMLS 73:1859-1870. DOI: 10.1007/s00018-016-2156-7.

Csárdi G., Nepusz T. 2006. The igraph software package for complex network research. InterJournal Complex Systems.

Dayalu P., Albin RL. 2015. Huntington Disease: Pathogenesis and Treatment. Neurologic Clinics 33:101114. DOI: $10.1016 /$ j.ncl.2014.09.003.

Downward J. 2003. Targeting RAS signalling pathways in cancer therapy. Nature Reviews Cancer 3:1122. DOI: $10.1038 / \mathrm{nrc} 969$.

Eijkelenboom A., Burgering BMT. 2013. FOXOs: signalling integrators for homeostasis maintenance. Nature Reviews Molecular Cell Biology 14:83-97. DOI: 10.1038/nrm3507.

Elia I., Broekaert D., Christen S., Boon R., Radaelli E., Orth MF., Verfaillie C., Grünewald TGP., Fendt S-M. 2017. Proline metabolism supports metastasis formation and could be inhibited to selectively target metastasizing cancer cells. Nature Communications 8:ncomms15267. DOI: 10.1038/ncomms15267. 
804 Fajardo AM., Piazza GA., Tinsley HN. 2014. The Role of Cyclic Nucleotide Signaling Pathways in Cancer:

805 Targets for Prevention and Treatment. Cancers 6:436-458. DOI: 10.3390/cancers6010436.

806 Flintoft L. 2004. Rewiring the network. Nature Reviews Genetics 5:808-808. DOI: 10.1038/nrg1476.

807 Franchini M., Mannucci PM. 2012. Past, present and future of hemophilia: a narrative review. Orphanet $808 \quad$ Journal of Rare Diseases 7:24. DOI: 10.1186/1750-1172-7-24.

809 Fu Z., Tindall DJ. 2008. FOXOs, cancer and regulation of apoptosis. Oncogene 27:2312-2319. DOI:

$810 \quad 10.1038 /$ onc.2008.24.

811 de la Fuente A. 2010. From 'differential expression' to 'differential networking' - identification of

812 dysfunctional regulatory networks in diseases. Trends in Genetics 26:326-333. DOI:

$813 \quad$ 10.1016/j.tig.2010.05.001.

814 Geier A. 2014. Hepatitis B virus: The "metabolovirus" highjacks cholesterol and bile acid metabolism.

$815 \quad$ Hepatology 60:1458-1460. DOI: 10.1002/hep.27224.

816 Goeman JJ., Bühlmann P. 2007. Analyzing gene expression data in terms of gene sets: methodological

817 issues. Bioinformatics (Oxford, England) 23:980-987. DOI: 10.1093/bioinformatics/btm051.

818 Golubovskaya VM., Kweh FA., Cance WG. 2009. Focal adhesion kinase and cancer. Histology and

819 Histopathology 24:503-510. DOI: 10.14670/HH-24.503.

820 Goossens N., Hoshida Y. 2015. Hepatitis C virus-induced hepatocellular carcinoma. Clinical and

821 Molecular Hepatology 21:105-114. DOI: 10.3350/cmh.2015.21.2.105.

822 Gross DN., van den Heuvel APJ., Birnbaum MJ. 2008. The role of FoxO in the regulation of metabolism.

823 Oncogene 27:2320-2336. DOI: 10.1038/onc.2008.25.

824 Gruol DL., Parsons KL. 1996. Chronic alcohol reduces calcium signaling elicited by glutamate receptor 825 stimulation in developing cerebellar neurons. Brain Research 728:166-174. DOI: 10.1016/0006826 8993(96)00185-0. 
827 Guertin DA., Sabatini DM. 2007. Defining the Role of mTOR in Cancer. Cancer Cell 12:9-22. DOI:

828

829

830

831

832

833

834

835

836

837

838

839

840

841

842

843

844

845

846

847

848

849

850

10.1016/j.ccr.2007.05.008.

Hautbergue GM. 2017. RNA Nuclear Export: From Neurological Disorders to Cancer. Advances in Experimental Medicine and Biology 1007:89-109. DOI: 10.1007/978-3-319-60733-7_6.

Hindorff LA., Gillanders EM., Manolio TA. 2011. Genetic architecture of cancer and other complex diseases: lessons learned and future directions. Carcinogenesis 32:945-954. DOI: 10.1093/carcin/bgr056.

Hoshida Y., Fuchs BC., Bardeesy N., Baumert TF., Chung RT. 2014. Pathogenesis and prevention of hepatitis C virus-induced hepatocellular carcinoma. Journal of Hepatology 61:S79-90. DOI: 10.1016/j.jhep.2014.07.010.

Hou JP., Ma J. 2014. DawnRank: discovering personalized driver genes in cancer. Genome Medicine 6:56. DOI: $10.1186 / \mathrm{s} 13073-014-0056-8$.

Hudson NJ., Reverter A., Dalrymple BP. 2009. A differential wiring analysis of expression data correctly identifies the gene containing the causal mutation. PLoS computational biology 5:e1000382. DOI: 10.1371/journal.pcbi.1000382.

Hynes NE., Lane HA. 2005. ERBB receptors and cancer: the complexity of targeted inhibitors. Nature Reviews Cancer 5:341-354. DOI: 10.1038/nrc1609.

Ideker T., Ozier O., Schwikowski B., Siegel AF. 2002. Discovering regulatory and signalling circuits in molecular interaction networks. Bioinformatics (Oxford, England) 18 Suppl 1:S233-240.

lozzo RV., Sanderson RD. 2011. Proteoglycans in cancer biology, tumour microenvironment and angiogenesis. Journal of Cellular and Molecular Medicine 15:1013-1031. DOI: 10.1111/j.15824934.2010.01236.x.

Kanehisa M., Goto S. 2000. KEGG: Kyoto Encyclopedia of Genes and Genomes. Nucleic Acids Research 28:27-30. 
851 Khanin R., Wit E. 2006. How scale-free are biological networks. Journal of Computational Biology: A 852 Journal of Computational Molecular Cell Biology 13:810-818. DOI: 10.1089/cmb.2006.13.810.

853 Khatri P., Sirota M., Butte AJ. 2012. Ten years of pathway analysis: current approaches and outstanding 854 challenges. PLoS computational biology 8:e1002375. DOI: 10.1371/journal.pcbi.1002375.

855 Kim K., Kim KH., Cheong J. 2010. Hepatitis B Virus X Protein Impairs Hepatic Insulin Signaling Through 856 Degradation of IRS1 and Induction of SOCS3. PLOS ONE 5. DOI: 10.1371/journal.pone.0008649.

857 Kimmel C., Visweswaran S. 2013. An algorithm for network-based gene prioritization that encodes 858 knowledge both in nodes and in links. PloS One 8:e79564. DOI: 10.1371/journal.pone.0079564.

859 Klauer AA., van Hoof A. 2012. Degradation of mRNAs that lack a stop codon: A decade of nonstop 860 progress. Wiley interdisciplinary reviews. RNA 3:649-660. DOI: 10.1002/wrna.1124.

861

862

863

864

865

866

867

868

869

870

871

872

873

Knight T., Irving JAE. 2014. Ras/Raf/MEK/ERK Pathway Activation in Childhood Acute Lymphoblastic Leukemia and Its Therapeutic Targeting. Frontiers in Oncology 4:160. DOI: 10.3389/fonc.2014.00160.

Kostka D., Spang R. 2004. Finding disease specific alterations in the co-expression of genes. Bioinformatics (Oxford, England) 20 Suppl 1:i194-199. DOI: 10.1093/bioinformatics/bth909.

Lai Y., Wu B., Chen L., Zhao H. 2004. A statistical method for identifying differential gene-gene coexpression patterns. Bioinformatics (Oxford, England) 20:3146-3155. DOI: 10.1093/bioinformatics/bth379.

Langfelder P., Luo R., Oldham MC., Horvath S. 2011. Is my network module preserved and reproducible? PLoS computational biology 7:e1001057. DOI: 10.1371/journal.pcbi.1001057.

Leonardson AS., Zhu J., Chen Y., Wang K., Lamb JR., Reitman M., Emilsson V., Schadt EE. 2010. The effect of food intake on gene expression in human peripheral blood. Human Molecular Genetics 19:159-169. DOI: 10.1093/hmg/ddp476. 
874 Li D., Li J., Guo Y. 2014. Genome-wide association study knowledge-driven pathway analysis of alcohol 875 dependence implicates the calcium signaling pathway. Chinese Medical Journal 127:2229-2235. 876 Lind DS. 2004. Arginine and Cancer. The Journal of Nutrition 134:2837S-2841S.

877 Lindeboom RGH., Supek F., Lehner B. 2016. The rules and impact of nonsense-mediated mRNA decay in 878 human cancers. Nature genetics 48:1112-1118. DOI: 10.1038/ng.3664.

879 Liu Z., Tian Y., Machida K., Lai MMC., Luo G., Foung SKH., Ou JJ. 2012. Transient Activation of the PI3K-

880

881

882

883

884

885

886

887

888

889

890

891

892

893

894

895

896

AKT Pathway by Hepatitis C Virus to Enhance Viral Entry. Journal of Biological Chemistry 287:41922-41930. DOI: 10.1074/jbc.M112.414789.

Liu B-H., Yu H., Tu K., Li C., Li Y-X., Li Y-Y. 2010. DCGL: an R package for identifying differentially coexpressed genes and links from gene expression microarray data. Bioinformatics (Oxford, England) 26:2637-2638. DOI: 10.1093/bioinformatics/btq471.

May P., May E. 1999. Twenty years of p53 research: structural and functional aspects of the p53 protein. Oncogene 18:7621-7636. DOI: 10.1038/sj.onc.1203285.

Mitrea C., Taghavi Z., Bokanizad B., Hanoudi S., Tagett R., Donato M., Voichiţa C., Drăghici S. 2013. Methods and approaches in the topology-based analysis of biological pathways. Frontiers in Physiology 4:278. DOI: 10.3389/fphys.2013.00278.

Molloy NH., Read DE., Gorman AM. 2011. Nerve Growth Factor in Cancer Cell Death and Survival. Cancers 3:510-530. DOI: 10.3390/cancers3010510.

Morrison JL., Breitling R., Higham DJ., Gilbert DR. 2005. GeneRank: using search engine technology for the analysis of microarray experiments. BMC bioinformatics 6:233. DOI: 10.1186/1471-2105-6233.

Morshed Osmani G., Rahman S. 2018. Using Google's PageRank Algorithm to Identify Important Attributes of Genes. 
897 Odibat O., Reddy CK. 2012. Ranking differential hubs in gene co-expression networks. Journal of Bioinformatics and Computational Biology 10:1240002. DOI: 10.1142/S0219720012400021.

899

900

901

902

903

904

905

906

907

908

909

910

911

912

913

914

915

916

917

918

919

920

Oehler N., Volz T., Bhadra OD., Kah J., Allweiss L., Giersch K., Bierwolf J., Riecken K., Pollok JM., Lohse AW., Fehse B., Petersen J., Urban S., Lütgehetmann M., Heeren J., Dandri M. 2014. Binding of hepatitis B virus to its cellular receptor alters the expression profile of genes of bile acid metabolism. Hepatology (Baltimore, Md.) 60:1483-1493. DOI: 10.1002/hep.27159.

Page L., Brin S., Motwani R., Winograd T. 1999. The PageRank Citation Ranking: Bringing Order to the Web.

Pan D. 2010. The Hippo Signaling Pathway in Development and Cancer. Developmental cell 19:491-505. DOI: 10.1016/j.devcel.2010.09.011.

Pedroche F., Moreno F., González A., Valencia A. 2013. Leadership groups on Social Network Sites based on Personalized PageRank. Mathematical and Computer Modelling 57:1891-1896. DOI: 10.1016/j.mcm.2011.12.026.

Pópulo H., Lopes JM., Soares P. 2012. The mTOR signalling pathway in human cancer. International Journal of Molecular Sciences 13:1886-1918. DOI: 10.3390/ijms13021886.

Porta C., Paglino C., Mosca A. 2014. Targeting PI3K/Akt/mTOR Signaling in Cancer. Frontiers in Oncology 4:64. DOI: 10.3389/fonc.2014.00064.

Rahmatallah Y., Emmert-Streib F., Glazko G. 2014. Gene Sets Net Correlations Analysis (GSNCA): a multivariate differential coexpression test for gene sets. Bioinformatics (Oxford, England) 30:360-368. DOI: 10.1093/bioinformatics/btt687.

Ramsahai E., Walkins K., Tripathi V., John M. 2017. The use of gene interaction networks to improve the identification of cancer driver genes. PeerJ 5:e2568. DOI: 10.7717/peerj.2568.

Reverter A., Ingham A., Lehnert SA., Tan S-H., Wang Y., Ratnakumar A., Dalrymple BP. 2006. Simultaneous identification of differential gene expression and connectivity in inflammation, 
921

922

923

924

925

926

927

928

929

930

931

932

933

934

935

936

937

938

939

940

941

942

943

944

adipogenesis and cancer. Bioinformatics (Oxford, England) 22:2396-2404. DOI:

10.1093/bioinformatics/btl392.

Rhinn H., Fujita R., Qiang L., Cheng R., Lee JH., Abeliovich A. 2013. Integrative genomics identifies APOE $\varepsilon 4$ effectors in Alzheimer's disease. Nature 500:45-50. DOI: 10.1038/nature12415.

Santarpia L., Lippman SM., El-Naggar AK. 2012. Targeting the MAPK-RAS-RAF signaling pathway in cancer therapy. Expert Opinion on Therapeutic Targets 16:103-119. DOI: 10.1517/14728222.2011.645805.

Sanyal AJ., Yoon SK., Lencioni R. 2010. The etiology of hepatocellular carcinoma and consequences for treatment. The Oncologist 15 Suppl 4:14-22. DOI: 10.1634/theoncologist.2010-S4-14.

Saucedo L., Edgar BA. 2007. Filling out the Hippo pathway. Nature Reviews Molecular Cell Biology 8:613-621. DOI: 10.1038/nrm2221.

Schmidt M., de Mattos SF., van der Horst A., Klompmaker R., Kops GJPL., Lam EW-F., Burgering BMT., Medema RH. 2002. Cell Cycle Inhibition by FoxO Forkhead Transcription Factors Involves Downregulation of Cyclin D. Molecular and Cellular Biology 22:7842-7852. DOI: 10.1128/MCB.22.22.7842-7852.2002.

Schork NJ. 1997. Genetics of complex disease: approaches, problems, and solutions. American Journal of Respiratory and Critical Care Medicine 156:S103-109. DOI: 10.1164/ajrccm.156.4.12-tac-5.

Schreiber G., Walter MR. 2010. Cytokine receptor interactions as drug targets. Current opinion in chemical biology 14:511-519. DOI: 10.1016/j.cbpa.2010.06.165.

Seshacharyulu P., Ponnusamy MP., Haridas D., Jain M., Ganti A., Batra SK. 2012. Targeting the EGFR signaling pathway in cancer therapy. Expert Opinion on Therapeutic Targets 16:15-31. DOI: 10.1517/14728222.2011.648617.

Sherr CJ., McCormick F. 2002. The RB and p53 pathways in cancer. Cancer Cell 2:103-112. DOI: 10.1016/S1535-6108(02)00102-2. 
945 Shi Q., Hoffman B., Liu Q. 2016. PI3K-Akt signaling pathway upregulates hepatitis C virus RNA translation

946 through the activation of SREBPs. Virology 490:99-108. DOI: 10.1016/j.virol.2016.01.012.

947 Siddiqui N., Borden KLB. 2012. mRNA export and cancer. Wiley interdisciplinary reviews. RNA 3:13-25.

$948 \quad$ DOI: 10.1002/wrna.101.

949 Simms CL., Yan LL., Zaher HS. 2017. Ribosome Collision Is Critical for Quality Control during No-Go Decay. $950 \quad$ Molecular Cell 68:361-373.e5. DOI: 10.1016/j.molcel.2017.08.019.

951 Spangler JB., Moraga I., Mendoza JL., Garcia KC. 2015. Insights into Cytokine-Receptor Interactions from

952 Cytokine Engineering. Annual review of immunology 33:139-167. DOI: 10.1146/annurev-

953 immunol-032713-120211.

954 Stegh AH. 2012. Targeting the p53 signaling pathway in cancer therapy - the promises, challenges and 955 perils. Expert Opinion on Therapeutic Targets 16:67-83. DOI: 10.1517/14728222.2011.643299.

956 Stuart JM., Segal E., Koller D., Kim SK. 2003. A gene-coexpression network for global discovery of 957 conserved genetic modules. Science (New York, N.Y.) 302:249-255. DOI:

958 10.1126/science.1087447.

Subramanian A., Tamayo P., Mootha VK., Mukherjee S., Ebert BL., Gillette MA., Paulovich A., Pomeroy 960 SL., Golub TR., Lander ES., Mesirov JP. 2005. Gene set enrichment analysis: a knowledge-based approach for interpreting genome-wide expression profiles. Proceedings of the National Academy of Sciences of the United States of America 102:15545-15550. DOI:

963 10.1073/pnas.0506580102.

964

Sui X., Jin L., Huang X., Geng S., He C., Hu X. 2011. p53 signaling and autophagy in cancer: A revolutionary strategy could be developed for cancer treatment. Autophagy 7:565-571. DOI:

966 10.4161/auto.7.6.14073.

967

Tai Y-L., Chen L-C., Shen T-L. 2015. Emerging roles of focal adhesion kinase in cancer. BioMed Research 968 International 2015:690690. DOI: 10.1155/2015/690690. 
969 Tarca AL., Draghici S., Khatri P., Hassan SS., Mittal P., Kim J-S., Kim CJ., Kusanovic JP., Romero R. 2009. A 970 novel signaling pathway impact analysis. Bioinformatics (Oxford, England) 25:75-82. DOI:

$971 \quad$ 10.1093/bioinformatics/btn577.

972 Tavazoie S., Hughes JD., Campbell MJ., Cho RJ., Church GM. 1999. Systematic determination of genetic 973 network architecture. Nature Genetics 22:281-285. DOI: 10.1038/10343.

974 Taylor IW., Linding R., Warde-Farley D., Liu Y., Pesquita C., Faria D., Bull S., Pawson T., Morris Q., Wrana

975 JL. 2009. Dynamic modularity in protein interaction networks predicts breast cancer outcome.

976 Nature Biotechnology 27:199-204. DOI: 10.1038/nbt.1522.

977 Tesson BM., Breitling R., Jansen RC. 2010. DiffCoEx: a simple and sensitive method to find differentially

978 coexpressed gene modules. BMC Bioinformatics 11:497. DOI: 10.1186/1471-2105-11-497.

979

Villanueva A., Chiang DY., Newell P., Peix J., Thung S., Alsinet C., Tovar V., Roayaie S., Minguez B., Sole

980

981

982

983

984

985 M., Battiston C., Van Laarhoven S., Fiel MI., Di Feo A., Hoshida Y., Yea S., Toffanin S., Ramos A., Martignetti JA., Mazzaferro V., Bruix J., Waxman S., Schwartz M., Meyerson M., Friedman SL., Llovet JM. 2008. Pivotal role of mTOR signaling in hepatocellular carcinoma. Gastroenterology 135:1972-1983, 1983.e1-11. DOI: 10.1053/j.gastro.2008.08.008.

Vojtek AB., Der CJ. 1998. Increasing complexity of the Ras signaling pathway. The Journal of Biological Chemistry 273:19925-19928.

986

987

988

989

990

991

992

Walker FO. 2007. Huntington's disease. The Lancet 369:218-228. DOI: 10.1016/S0140-6736(07)60111-1.

Wang W., Pan Q., Fuhler GM., Smits R., Peppelenbosch MP. 2017. Action and function of Wnt/ $\beta$-catenin signaling in the progression from chronic hepatitis $C$ to hepatocellular carcinoma. Journal of Gastroenterology 52:419-431. DOI: 10.1007/s00535-016-1299-5.

Wang C., Wang X., Gong G., Ben Q., Qiu W., Chen Y., Li G., Wang L. 2012. Increased risk of hepatocellular carcinoma in patients with diabetes mellitus: a systematic review and meta-analysis of cohort studies. International Journal of Cancer 130:1639-1648. DOI: 10.1002/ijc.26165. 
993 Wang R., Zhang W., Deng H., Wang N., Miao Q., Zhao X. 2013. Discover Community Leader in Social $994 \quad$ Network with PageRank. In: Advances in Swarm Intelligence. Springer, Berlin, Heidelberg, 154995 162. DOI: 10.1007/978-3-642-38715-9_19.

996

997

998

999

1000

1001

1002

1003

1004

1005

1006

1007

1008

1009

1010

1011

1012

1013

1014

1015

1016

Watson M. 2006. CoXpress: differential co-expression in gene expression data. BMC bioinformatics 7:509. DOI: 10.1186/1471-2105-7-509.

Weirauch MT. 2011. Gene Coexpression Networks for the Analysis of DNA Microarray Data. In: Dehmer thias, Emmert-Streib F, Graber A, Salvador A eds. Applied Statistics for Network Biology. WileyVCH Verlag GmbH \& Co. KGaA, 215-250. DOI: 10.1002/9783527638079.ch11.

Winter C., Kristiansen G., Kersting S., Roy J., Aust D., Knösel T., Rümmele P., Jahnke B., Hentrich V., Rückert F., Niedergethmann M., Weichert W., Bahra M., Schlitt HJ., Settmacher U., Friess H., Büchler M., Saeger H-D., Schroeder M., Pilarsky C., Grützmann R. 2012. Google goes cancer: improving outcome prediction for cancer patients by network-based ranking of marker genes. PLoS computational biology 8:e1002511. DOI: 10.1371/journal.pcbi.1002511.

Zeng X., Zhao J., Wu X., Shi H., Liu W., Cui B., Yang L., Ding X., Song P. 2016. PageRank analysis reveals topologically expressed genes correspond to psoriasis and their functions are associated with apoptosis resistance. Molecular Medicine Reports 13:3969-3976. DOI: 10.3892/mmr.2016.4999.

Zhang B., Li H., Riggins RB., Zhan M., Xuan J., Zhang Z., Hoffman EP., Clarke R., Wang Y. 2009. Differential dependency network analysis to identify condition-specific topological changes in biological networks. Bioinformatics (Oxford, England) 25:526-532. DOI: 10.1093/bioinformatics/btn660.

Zhang Y-L., Wang R-C., Cheng K., Ring BZ., Su L. 2017. Roles of Rap1 signaling in tumor cell migration and invasion. Cancer Biology \& Medicine 14:90-99. DOI: 10.20892/j.issn.2095-3941.2016.0086.

Zhou L., Huang Y., Li J., Wang Z. 2010. The mTOR pathway is associated with the poor prognosis of human hepatocellular carcinoma. Medical Oncology (Northwood, London, England) 27:255-261. DOI: $10.1007 /$ s12032-009-9201-4. 
1017 Zhu X., Gerstein M., Snyder M. 2007. Getting connected: analysis and principles of biological networks.

1018 Genes \& Development 21:1010-1024. DOI: 10.1101/gad.1528707.

1019 
Figure 1

Overview of the PoTRA method.

PageRank Analysis for Genes for normal and cancer

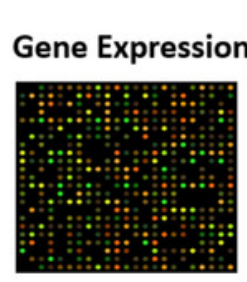

Step 1

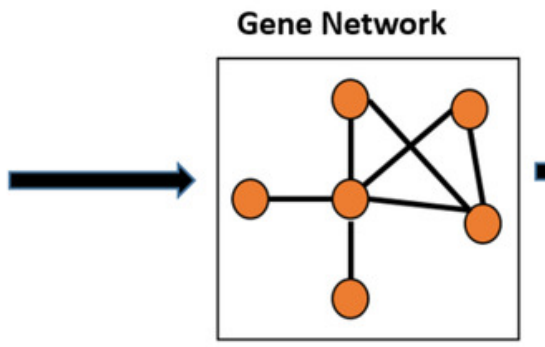

Significant Pathways of Topological Rank Analysis

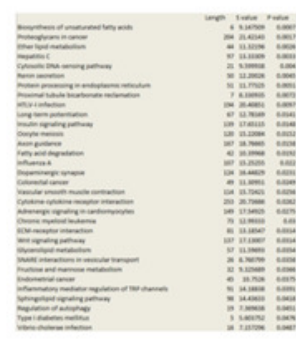

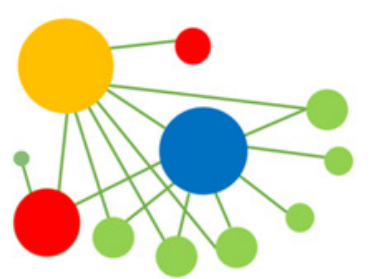

Normal

Step 3
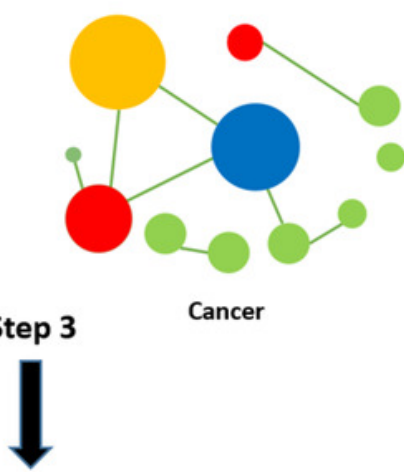

Detect pathways with the altered number of hub genes or distributions between normal and cancer

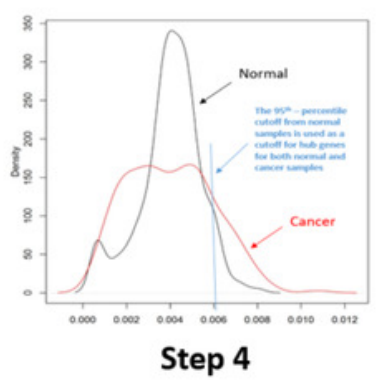




\section{Figure 2}

The topological rank analysis for each gene within a pathway.

For genes within a specified pathway, according to Step 2, we construct a corresponding gene co-expression network for normal and cancer, separately. Then we apply the PageRank method to obtain the topological importance of each gene for normal and cancer, separately. PR(gene i $)_{\text {normal }}$ represents the PageRank score of the gene i for normal samples, while PR(gene i) cancer represents the PageRank score of the gene i for cancer samples.

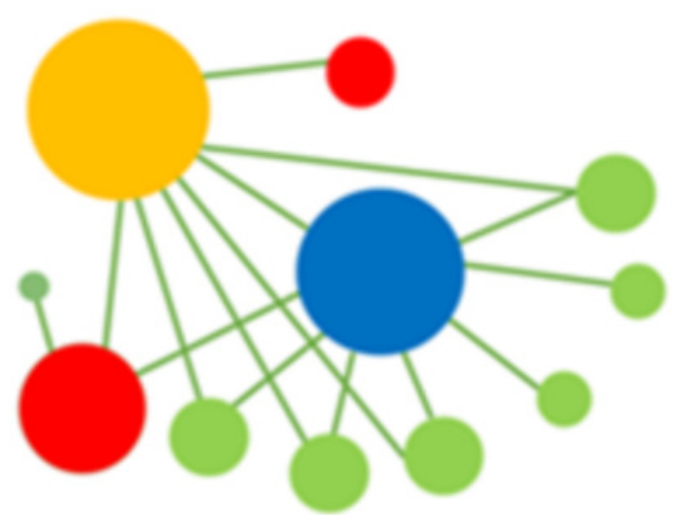

Normal a. PR(gene i) normal

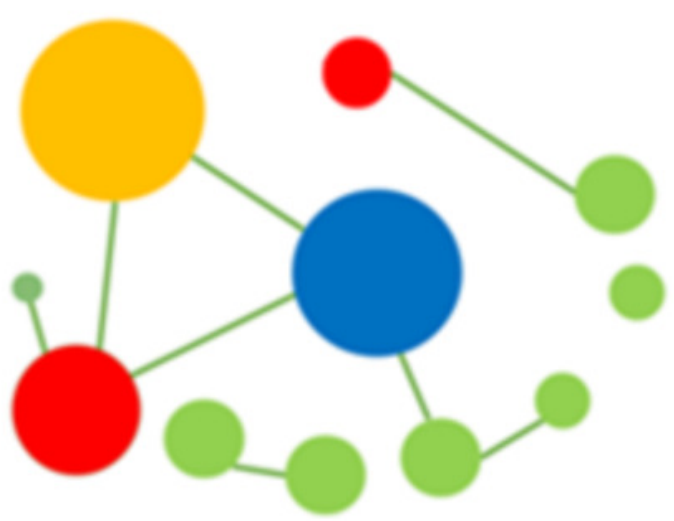

Cancer 


\section{Figure 3}

The kernel density distribution of PageRank scores of genes in "MAPK signaling pathway".

The red line shows the kernel density distribution of PageRank scores for cancer and the black one is for normal samples. Note that the mean for the two distributions is the same, i.e., mean $=1 / N=0.004$, where $N=250$ is the number of genes in the "MAPK signaling pathway" pathway. We use the 95th-percentile cutoff $(=0.006035)$ of the kernel distribution in normal samples as cutoff for hub genes for both normal and cancer samples. 


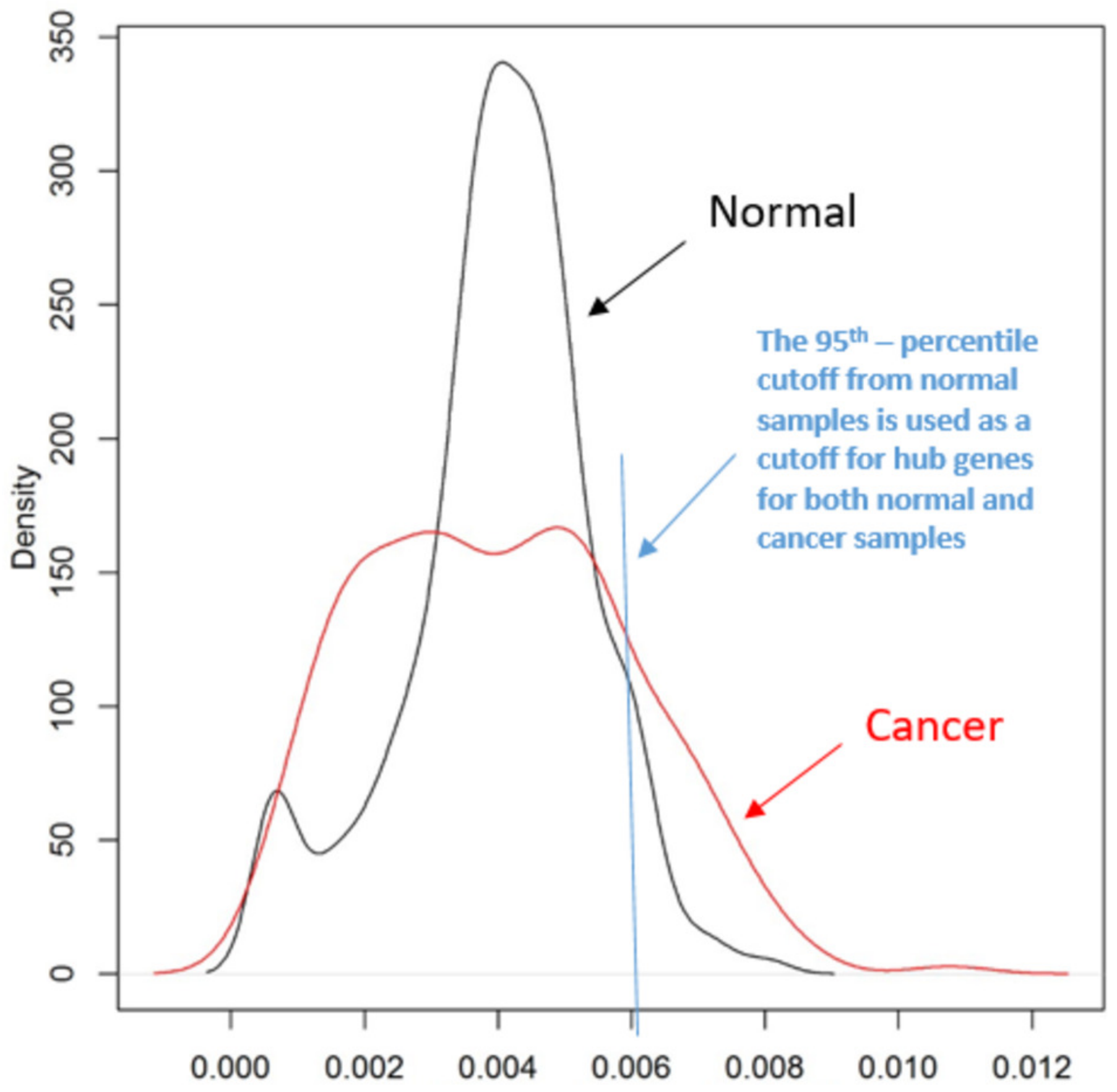




\section{Table $\mathbf{1}$ (on next page)}

Contingency table for Fisher's exact test.

We use the $95^{\text {th }}$ percentile of the distribution (one-tail) in normal samples as cutoff value for hub genes for both normal and cancer samples. The value "a" represents the number of genes whose PageRank scores are below the cutoff value for normal samples. The value " $b$ " represents the number of genes whose PageRank scores are above the cutoff value for normal samples. The values " $c$ " and " $d$ " are the corresponding values for cancer. We use Fisher's exact test to assess if the number of hub genes is significantly different between normal and cancer. 
Number of non-hub genes Number of hub genes

Total

Normal

$\mathbf{a}$

b

d

$b+d$ $\mathbf{a}+\mathbf{b}$

$\mathbf{c}+\mathbf{d}$

$\mathbf{a}+\mathbf{b}+\mathbf{c}+\mathbf{d}$

Table 1. Contingency table for Fisher's exact test. We use the $95^{\text {th }}$ percentile of the distribution (one-tail) in normal samples as cutoff value for hub genes for both normal and cancer samples. The value "a" represents the number of genes whose PageRank scores are below the cutoff value for normal samples. The value " $b$ " represents the number of genes whose PageRank scores are above the cutoff value for normal samples. The values "c" and " $\mathrm{d}$ " are the corresponding values for cancer. We use Fisher's exact test to assess if the number of hub genes is significantly different between normal and cancer. 


\section{Table 2 (on next page)}

The "MAPK signaling pathway" pathway identified by PoTRA for HCC using Fisher's exact test.

The $\mathrm{P}$ value is adjusted by False Discovery Rate (FDR). 


$\begin{array}{llllll}\text { Gene } & \text { \# of } & \text { \# of } & \text { \# of hub } & \text { \# of hub } & \text { Adjusted } \\ \text { Count(L) } & \text { edges_normal } & \text { edges_cancer } & \text { genes_normal } & \text { genes_cancer } & \text { P-value }\end{array}$

MAPK

signaling

pathway

250

14005

5170

13

40

0.0158

Table 2. The "MAPK signaling pathway" pathway identified by PoTRA for HCC using Fisher's exact test. The P value is adjusted by False Discovery Rate (FDR). 


\section{Table 3(on next page)}

The significant KEGG pathways identified by PoTRA for HCC using Fisher's exact test.

FDR adjusted P-values are below 0.05 . 


$$
\begin{gathered}
\text { \# of edges } \# \text { of hub \# of hub } \\
\text { Gene edges_- genes_ genes_ Adjusted } \\
\text { Count(L) normal cancer normal cancer P-value }
\end{gathered}
$$

Gene edges___ genes_ genes_ Adjusted

1 Pathways in cancer 310 249249136

48

0.0081

MAPK signaling

2 pathway 25

3 Breast cancer

$\begin{array}{llllll}143 & 3589 & 1175 & 8 & 29 & 0.0278\end{array}$

Table 3. The significant KEGG pathways identified by PoTRA for HCC using Fisher's exact test. FDR adjusted P-values are below 0.05 . 
Table 4(on next page)

The "MAPK signaling pathway" pathway identified by PoTRA for HCC using the Kolmogorov-Smirnov test.

The $\mathrm{P}$ value is adjusted by False Discovery Rate (FDR). 
Gene Count(L) \# of edges_normal \# of edges_cancer Adjusted P-value

MAPK signaling

pathway

250

14005

5170

0.0278423

Table 4. The "MAPK signaling pathway" pathway identified by PoTRA for HCC using the Kolmogorov-Smirnov test. The $\mathrm{P}$ value is adjusted by False Discovery Rate (FDR). 


\section{Table 5 (on next page)}

The significant KEGG pathways identified by PoTRA for HCC using the Kolmogorov-Smirnov test.

FDR adjusted P-values are below 0.05 . 
Gene

Count(L) \# of edges_normal \# of edges_cancer Adjusted P-value

1 RNA transport

133

7168

5343

6.72E-08

2 mRNA surveillance pathway

70

1867

1252

0.023328272

3 MAPK signaling pathway

250

14005

5170

0.027842298

Table 5. The significant KEGG pathways identified by PoTRA for HCC using the Kolmogorov-Smirnov test. FDR adjusted P-values are below 0.05. 


\section{Table 6(on next page)}

The significant KEGG pathways identified by PoTRA for hepatitis B-induced HCC using Fisher's exact test.

FDR adjusted P-values are below 0.05 . 


\begin{tabular}{|c|c|c|c|c|c|c|}
\hline & $\begin{array}{l}\text { Gene } \\
\text { Count(L) }\end{array}$ & $\begin{array}{l}\text { \# of } \\
\text { edges__ } \\
\text { normal }\end{array}$ & $\begin{array}{l}\text { \# of } \\
\text { edges_- } \\
\text { cancer }\end{array}$ & $\begin{array}{l}\text { \# of hub } \\
\text { genes_- } \\
\text { normal }\end{array}$ & $\begin{array}{l}\text { \# of hub } \\
\text { genes_- } \\
\text { cancer }\end{array}$ & $\begin{array}{l}\text { Adjusted } \\
\text { P-value }\end{array}$ \\
\hline 1 Insulin signaling pathway & 139 & 2692 & 958 & 7 & 34 & 0.0007 \\
\hline 2 Pathways in cancer & 310 & 11194 & 3792 & 16 & 52 & 0.0007 \\
\hline 3 Hippo signaling pathway & 151 & 2836 & 970 & 8 & 31 & 0.0072 \\
\hline 4HTLV-I infection & 194 & 5518 & 2080 & 10 & 35 & 0.0072 \\
\hline $\begin{array}{l}\text { Neurotrophin signaling } \\
5 \text { pathway }\end{array}$ & 117 & 2441 & 895 & 6 & 25 & 0.0195 \\
\hline $6 \mathrm{mTOR}$ signaling pathway & 144 & 3410 & 832 & 8 & 28 & 0.0240 \\
\hline $\begin{array}{l}\text { Epstein-Barr virus } \\
7 \text { infection }\end{array}$ & 85 & 1524 & 435 & 5 & 21 & 0.0353 \\
\hline 8 Hepatitis B & 134 & 2708 & 828 & 7 & 25 & 0.0353 \\
\hline
\end{tabular}

Table 6. The significant KEGG pathways identified by PoTRA for hepatitis B-induced HCC using Fisher's exact test. FDR adjusted P-values are below 0.05. 


\section{Table 7 (on next page)}

The significant KEGG pathways identified by PoTRA for hepatitis C-induced HCC using Fisher's exact test.

FDR adjusted P-values are below 0.05 . 


$\begin{array}{llllll} & \text { \# of } & \text { \# of } & \text { \# of hub } & \text { \# of hub } & \\ \text { Gene } & \text { edges_ } & \text { edges_} & \text { genes__ } & \text { genes_ } & \text { Adjusted } \\ \text { Count(L) } & \text { normal } & \text { cancer } & \text { normal } & \text { cancer } & \text { P-value }\end{array}$

1 Pathways in cancer

310

$22253 \quad 7791$

16

2.89E-

PI3K-Akt signaling

2 pathway

340

$19901 \quad 6594$

17

62

06

3 MAPK signaling pathway

25

4 Proteoglycans in cancer

204

$11986 \quad 4168$

13

$9815 \quad 3642$

11

$2.89 \mathrm{E}-$

5 Rap1 signaling pathway

208

$8294 \quad 3587$

11

06

Adrenergic signaling in

6 cardiomyocytes

149

$3594 \quad 1355$

8

$27 \quad 0.0372$

7 cAMP signaling pathway

196

$5106 \quad 2493$

10

$30 \quad 0.0372$

8 Focal adhesion

203

10225

4656

11

$32 \quad 0.0372$

9 HTLV-I infection

194

9843

4030

10

$30 \quad 0.0372$

10 Ras signaling pathway

226

10098

3931

12

$33 \quad 0.0376$

11 FoxO signaling pathway

126

3391

1222

7

$24 \quad 0.0380$

12 Osteoclast differentiation

123

4418

1452

$24 \quad 0.0380$

13 ErbB signaling pathway

88

2128

814

20

0.0400

14Axon guidance

167

$6203 \quad 2705$

9

27

0.0433

Table 7. The significant KEGG pathways identified by PoTRA for hepatitis C-induced

HCC using Fisher's exact test. FDR adjusted P-values are below 0.05. 


\section{Table 8 (on next page)}

The significant KEGG pathways identified by PoTRA for alcohol-induced HCC using Fisher's exact test.

FDR adjusted P-values are below 0.05 . 


\begin{tabular}{|c|c|c|c|c|c|}
\hline & $\begin{array}{l}\text { \# of } \\
\text { edges }\end{array}$ & $\begin{array}{l}\text { \# of } \\
\text { edges_ }\end{array}$ & $\begin{array}{l}\text { \# of hub } \\
\text { genes }\end{array}$ & $\begin{array}{l}\text { \# of hub } \\
\text { genes }\end{array}$ & $\begin{array}{l}\text { Adjuste } \\
\mathrm{d}\end{array}$ \\
\hline (L) & normal & cancer & normal & cancer & $\mathrm{P}$-value \\
\hline
\end{tabular}

PI3K-Akt signaling 1 pathway

23928

8733

17

55

0.0006

MAPK signaling

2 pathway

252

14005

5767

13

46

0.0007

3 Pathways in cancer

310

$24924 \quad 10191$

16

$47 \quad 0.0043$

Table 8. The significant KEGG pathways identified by PoTRA for alcohol-induced HCC using Fisher's exact test. FDR adjusted P-values are below 0.05 . 
Table 9 (on next page)

The significant KEGG pathways identified by PoTRA for hepatitis C-induced HCC using the Kolmogorov-Smirnov test.

FDR adjusted P-values are below 0.05 . 
Gene

Count(L) \# of edges_normal \# of edges_cancer Adjusted P-value

1 RNA transport

2 Pathways in cancer

3 Proteoglycans in cancer

4 MAPK signaling pathway

PI3K-Akt signaling

5 pathway

6 HTLV-I infection
133

6877

22253

9815

11986

19901

340

194

9843

4001

3.33E-06

7791

0.0055638

3642

0.0055638

Table 9. The significant KEGG pathways identified by PoTRA for hepatitis $\mathrm{C}$-induced HCC using the Kolmogorov-Smirnov test. FDR adjusted P-values are below 0.05. 


\section{Table $\mathbf{1 0}$ (on next page)}

The significant KEGG pathways identified by PoTRA for alcohol-induced HCC using the Kolmogorov-Smirnov test.

FDR adjusted P-values are below 0.05 . 
Gene

Count(L) \# of edges_normal \# of edges_cancer Adjusted P-value

1 RNA transport

2 Pathways in cancer

$3 \mathrm{PI}$ KK-Akt signaling pathway

4MAPK signaling pathway

5 mRNA surveillance pathway
133

7168

24924

23928

14005

1867
4816

10191

8733

5767

1096
2.94E-08

0.0062832

0.0062832

0.01050039

0.01166414

Table 10. The significant KEGG pathways identified by PoTRA for alcohol-induced HCC using the Kolmogorov-Smirnov test. FDR adjusted P-values are below 0.05 . 


\section{Table $\mathbf{1 1}$ (on next page)}

The significant KEGG pathways identified by PoTRA for hepatitis B-induced HCC using the Kolmogorov-Smirnov test.

FDR adjusted P-values are below 0.05 . 
Gene

Count(L) \# of edges_normal \# of edges_cancer Adjusted P-value

Arginine and proline

1 metabolism

50

163

26

0.005412

Glyoxylate and dicarboxylate

2 metabolism

26

216

19

0.02765948

3 Primary bile acid biosynthesis

17

62

6

0.02765948

4 Insulin signaling pathway

139

2692

958

0.04057992

Vasopressin-regulated water

5 reabsorption

22

58

8

0.04057992

Table 11. The significant KEGG pathways identified by PoTRA for hepatitis B-induced HCC using the Kolmogorov-Smirnov test. FDR adjusted P-values are below 0.05. 


\section{Table $\mathbf{1 2}$ (on next page)}

The significant KEGG pathways identified by PoTRA for hepatitis C-induced HCC using the Fisher's exact test based on combined networks.

FDR adjusted P-values are below 0.05. E.comb.normal represents the number of edges in the combined network for normal samples, while E.comb.case is for cancer samples, respectively. 


$\begin{array}{llrrrr} & & \text { Gene Counts } & \text { E.comb.normal } & \text { E.comb.case } & \text { Adjusted P value } \\ 1 & \text { Epstein-Barr virus infection } & 85 & 131 & 43 & 0.0103488 \\ 2 & \text { p53 signaling pathway } & 68 & 57 & 20 & 0.0103488\end{array}$

Table 12. The significant KEGG pathways identified by PoTRA for hepatitis C-induced

HCC using the Fisher's exact test based on combined networks. FDR adjusted P-values are below 0.05. E.comb.normal represents the number of edges in the combined network for normal samples, while E.comb.case is for cancer samples, respectively. 


\section{Table $\mathbf{1 3}$ (on next page)}

The significant KEGG pathways identified by PoTRA for hepatitis B-induced HCC using the Fisher's exact test based on combined networks.

FDR adjusted P-values are below 0.05. E.comb.normal represents the number of edges in the combined network for normal samples, while E.comb.case is for cancer samples, respectively. 
Gene Counts E.comb.normal E.comb.case Adjusted P value

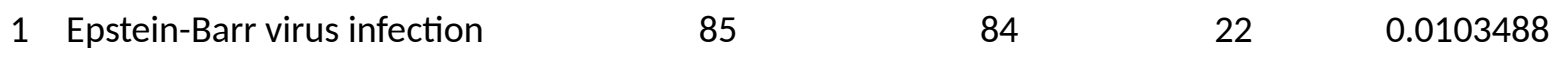

Table 13. The significant KEGG pathways identified by PoTRA for hepatitis B-induced

HCC using the Fisher's exact test based on combined networks. FDR adjusted P-values are

below 0.05. E.comb.normal represents the number of edges in the combined network for normal samples, while E.comb.case is for cancer samples, respectively. 